\title{
Loops in SU(2), Riemann Surfaces, and Factorization, $\mathbf{I}^{\star}$
}

\author{
Estelle BASOR ${ }^{\dagger}$ and Doug PICKRELL $\ddagger$ \\ $\dagger$ American Institute of Mathematics, 600 E. Brokaw Road, San Jose, CA 95112, USA \\ E-mail: ebasor@aimath.org \\ ‡ Mathematics Department, University of Arizona, Tucson, AZ 85721, USA \\ E-mail: pickrell@math.arizona.edu
}

Received October 24, 2015, in final form March 02, 2016; Published online March 08, 2016

http://dx.doi.org/10.3842/SIGMA.2016.025

\begin{abstract}
In previous work we showed that a loop $g: S^{1} \rightarrow \mathrm{SU}(2)$ has a triangular factorization if and only if the loop $g$ has a root subgroup factorization. In this paper we present generalizations in which the unit disk and its double, the sphere, are replaced by a based compact Riemann surface with boundary, and its double. One ingredient is the theory of generalized Fourier-Laurent expansions developed by Krichever and Novikov. We show that a $\mathrm{SU}(2)$ valued multiloop having an analogue of a root subgroup factorization satisfies the condition that the multiloop, viewed as a transition function, defines a semistable holomorphic $\operatorname{SL}(2, \mathbb{C})$ bundle. Additionally, for such a multiloop, there is a corresponding factorization for determinants associated to the spin Toeplitz operators defined by the multiloop.
\end{abstract}

Key words: loop group; factorization; Toeplitz operator; determinant

2010 Mathematics Subject Classification: 22E67; 47A68; 47B35

This paper is dedicated to Percy Deift and Craig Tracy, both of whom have contributed to the richness and beauty of mathematics and mathematical physics in so many ways. Both have created techniques and theories that have enhanced the understanding of fundamental problems and questions. And both with their enthusiasm and fearlessness, have encouraged, inspired, and motivated others to try to do the same.

The authors of this paper met in a summer meeting in 1984 in Laramie, Wyoming, where Craig was one of the principal speakers. One of the topics discussed informally at the meeting was determinants of Toeplitz operators, a subject where both Percy and Craig have made considerable contributions, in particular with the study of singular symbols and applications to statistical mechanics. This paper concerns some generalized Toeplitz determinant calculations for matrix-valued symbols, a topic that is a return to a long ago summer, yet one which is still of current interest.

\section{Introduction}

Suppose that $\Sigma$ is a connected compact Riemann surface with nonempty boundary $S$ (a disjoint union of circles). Let $\widehat{\Sigma}$ denote the double,

$$
\widehat{\Sigma}=\Sigma^{*} \circ \Sigma,
$$

${ }^{\star}$ This paper is a contribution to the Special Issue on Asymptotics and Universality in Random Matrices, Random Growth Processes, Integrable Systems and Statistical Physics in honor of Percy Deift and Craig Tracy. The full collection is available at http://www.emis.de/journals/SIGMA/Deift-Tracy.html 
where $\Sigma^{*}$ is the adjoint of $\Sigma$, i.e., the surface $\Sigma$ with the orientation reversed, and the composition is sewing along the common boundary $S$. Let $R$ denote the antiholomorphic involution (or reflection) fixing $S$.

The classical example is $\Sigma=D$, the closed unit disk. In this case $S=S^{1}, \widehat{\Sigma}$ is isomorphic to the Riemann sphere, and (in this realization) $R(z)=1 / z^{*}$, where $z^{*}=\bar{z}$, the complex conjugate. This example has the exceptional feature that there is a large automorphism group, $\operatorname{PSU}(1,1)$, acting by linear fractional transformations.

We now choose a basepoint, denoted by (0), in the interior of $\Sigma$, and we let $(\infty)$ denote the reflected basepoint for $\Sigma^{*}$. In the classical case, without loss of generality because of the $\operatorname{PSU}(1,1)$ symmetry, we can assume the basepoint is $z=0$. Given the data $(\Sigma,(0))$, following ideas of Krichever and Novikov, a reasonable function on $S$ has a 'linear triangular factorization'

$$
f=f_{-}+f_{0}+f_{+},
$$

where $f_{ \pm}$is holomorphic in the interior of $\Sigma\left(\Sigma^{*}\right.$, respectively), with appropriate boundary behavior, depending on the smoothness of $f, f_{+}((0))=0, f_{-}((\infty))=0$, and $f_{0}$ is the restriction to $S$ of a meromorphic function which belongs to a genus $(\widehat{\Sigma})+1$-dimensional complementary subspace, which we refer to as the vector space of zero modes (see Proposition 2.3). In the classical case $f_{0}$ is the zero mode for the Fourier series of $f$.

A holomorphic map $\mathfrak{z}: \widehat{\Sigma} \rightarrow \widehat{D}$ is said to be strictly equivariant if it satisfies

$$
\mathfrak{z}(R(q))=\frac{1}{\mathfrak{z}(q)^{*}}
$$

and maps $\Sigma$ to $D$ (and hence $\Sigma^{*}$ to $D^{*}$ ). When we refer to the classical case $(\Sigma=D)$, it will be understood that $\mathfrak{z}(z)=z$. For a function $f: U \subset \widehat{\Sigma} \rightarrow \mathcal{L}\left(\mathbb{C}^{N}\right)$, define $f^{*}(q)=f(R(q))^{*}$, where $(\cdot)^{*}$ is the Hermitian adjoint. If $f \in H^{0}(\Sigma)$ (i.e., a holomorphic function in some open neighborhood of $\Sigma$ ), then $f^{*} \in H^{0}\left(\Sigma^{*}\right)$. If $q \in S$, then $f^{*}(q)=f(q)^{*}$, the ordinary complex conjugate of $f(q)$.

Theorem 1.1. Suppose that $k_{1} \in C^{\infty}(S, \mathrm{SU}(2))$. Consider the following three conditions:

(I.1) $k_{1}$ is of the form

$$
k_{1}(z)=\left(\begin{array}{cc}
a(z) & b(z) \\
-b^{*}(z) & a^{*}(z)
\end{array}\right), \quad z \in S,
$$

where $a$ and $b$ are boundary values of holomorphic function in $\Sigma$ with $a((0))>0$, and $a$ and $b$ do not simultaneously vanish at a point in $\Sigma$.

(I.2) $k_{1}$ has a "root subgroup factorization" of the form

$$
k_{1}(z)=\lim _{n \rightarrow \infty} \mathbf{a}\left(\eta_{n}\right)\left(\begin{array}{cc}
1 & -\bar{\eta}_{n} \mathfrak{z}^{n} \\
\eta_{n} \mathfrak{z}^{-n} & 1
\end{array}\right) \cdots \mathbf{a}\left(\eta_{0}\right)\left(\begin{array}{cc}
1 & -\bar{\eta}_{0} \\
\eta_{0} & 1
\end{array}\right),
$$

for some rapidly decreasing sequence $\left\{\eta_{0}, \ldots, \eta_{n}, \ldots\right\}$ of complex numbers, and for some strictly equivariant function $\mathfrak{z}$ with $\mathfrak{z}((0))=0$.

(I.3) $k_{1}$ has a (multiplicative triangular) factorization of the form

$$
\left(\begin{array}{cc}
1 & 0 \\
y^{*}(z)+y_{0}(z) & 1
\end{array}\right)\left(\begin{array}{cc}
a_{1} & 0 \\
0 & a_{1}^{-1}
\end{array}\right)\left(\begin{array}{cc}
\alpha_{1}(z) & \beta_{1}(z) \\
\gamma_{1}(z) & \delta_{1}(z)
\end{array}\right)
$$

where $a_{1}>0$, the third factor is a $\mathrm{SL}(2, \mathbb{C})$-valued holomorphic function in $\Sigma$ which is unipotent upper triangular at $0, y=y_{+}$is holomorphic in $\Sigma$, and $y_{0}$ is a zero mode (as in (1.1)).

Then (I.2) implies (I.1) and (I.3) (with $\left.y_{0}=0\right)$, and (I.1) and (I.3) are equivalent. 
Similarly, consider $k_{2} \in C^{\infty}(S, \mathrm{SU}(2))$ and the following statements:

(II.1) $k_{2}$ is of the form

$$
k_{2}(z)=\left(\begin{array}{cc}
d^{*}(z) & -c^{*}(z) \\
c(z) & d(z)
\end{array}\right), \quad z \in S^{1}
$$

where $c$ and $d$ are boundary values of holomorphic functions in $\Sigma, c((0))=0$, and $d((0))>0$, and $c$ and $d$ do not simultaneously vanish at a point in $\Sigma$.

(II.2) $k_{2}$ has a "root subgroup factorization" of the form

$$
k_{2}(z)=\lim _{n \rightarrow \infty} \mathbf{a}\left(\zeta_{n}\right)\left(\begin{array}{cc}
1 & \zeta_{n \mathfrak{z}^{-n}} \\
-\bar{\zeta}_{n} \mathfrak{z}^{n} & 1
\end{array}\right) \cdots \mathbf{a}\left(\zeta_{1}\right)\left(\begin{array}{cc}
1 & \zeta_{1 \mathfrak{z}^{-1}} \\
-\bar{\zeta}_{1 \mathfrak{z}} & 1
\end{array}\right)
$$

for some rapidly decreasing sequence $\left\{\zeta_{1}, \ldots, \zeta_{n}, \ldots\right\}$ of complex numbers, and for some strictly equivariant $\mathfrak{z}$ with $\mathfrak{z}((0))=0$.

(II.3) $k_{2}$ has (triangular) factorization of the form

$$
\left(\begin{array}{cc}
1 & x^{*}(z)+x_{0}(z) \\
0 & 1
\end{array}\right)\left(\begin{array}{cc}
a_{2} & 0 \\
0 & a_{2}^{-1}
\end{array}\right)\left(\begin{array}{cc}
\alpha_{2}(z) & \beta_{2}(z) \\
\gamma_{2}(z) & \delta_{2}(z)
\end{array}\right)
$$

where $a_{2}>0$, the third factor is a $\mathrm{SL}(2, \mathbb{C})$-valued holomorphic function which is unipotent upper triangular at $(0), x=x_{+}$is holomorphic in $\Sigma$, and $x_{0}$ is a zero mode (as in (1.1)).

Then (II.2) implies (II.1) and (II.3) (with $x_{0}=0$ ), and (II.1) and (II.3) are equivalent.

\section{Remark 1.2.}

(a) In the classical case, all three conditions are equivalent (with $\mathfrak{z}=z$ ), and there are no constraints on $y^{*}$ or $x^{*}$ in (I.3) and (II.3), respectively (see [15]).

(b) In nonclassical cases there does not exist an equivariant function $\mathfrak{z}$ for which all three conditions are equivalent (because for example (II.2) implies $x_{0}=0$ in (II.3)). In this paper we will primarily work with the conditions (I.1) and (I.3), and (II.1) and (II.3).

(c) The second condition shows how to generate examples of loops with $y_{0}=0$ and $x_{0}=0$ in (I.3) and (II.3), respectively. We lack a method to generate transparent examples with $y_{0} \neq 0$ or $x_{0} \neq 0$. At this point we do not know whether there are constraints on the set of $x^{*}+x_{0}$ which occur in (II.3).

(d) Below we will explain why it is essential to generalize this theorem by allowing $a$ and $b$ $(c$ and $d$ ) to simultaneously vanish in $\Sigma$ (in a controlled way) in (I.1) ((II.1), respectively), and to allow $a_{1}\left(a_{2}\right)$ to be a function in (I.3) ((II.3), respectively). A tentative step in this direction is discussed in Section 3.1.

In the classical case a loop $g: S^{1} \rightarrow \mathrm{SU}(2)$ has a root subgroup factorization

$$
g=k_{1}^{*} \operatorname{diag}\left(e^{\chi}, e^{-\chi}\right) k_{2}
$$

if and only if $g$ has a triangular factorization, a generic condition, and these factorizations are unique (see [15]). In our more general context we ask similar questions: can we characterize the set of $g$ which have a factorization $g=k_{1}^{*} \operatorname{diag}\left(e^{\chi}, e^{-\chi}\right) k_{2}$, is the relevant condition generic, and is the factorization unique? An immediate obstacle is that, to our knowledge, there does not currently exist an analogue of the theory of triangular (or Birkhoff or Riemann-Hilbert) factorization for matrix valued multiloops in the context of this paper (see [18] for a survey of results in this direction, and [4] or [16, Chapter 8] for further background and history). Partly 
for this reason, we express what we know about these questions in terms of the holomorphic bundle defined by viewing a loop as a transition function. For example in the classical case, as observed by Grothendieck, a loop $g: S^{1} \rightarrow \mathrm{SL}(2, \mathbb{C})$ has a Riemann-Hilbert factorization $g=g_{-} g_{0} g_{+}$, where $g_{-} \in H^{0}\left(\Delta^{*}, \operatorname{SL}(2, \mathbb{C})\right), g_{0} \in \mathrm{SL}(2, \mathbb{C})$, and $g_{+} \in H^{0}(\Delta, \operatorname{SL}(2, \mathbb{C}))$, if and only if the corresponding holomorphic $\operatorname{SL}(2, \mathbb{C})$ bundle on $\mathbb{P}^{1}$ is trivial (see [7], or [16, Chapter 8]); in the case of the sphere, such a bundle is trivial if and only if it is semistable (for the definition and basic properties of semistability of principal bundles, see [17]; for an alternative exposition, which inspires some of the goals of our project, see [2]).

Theorem 1.3. Suppose $g \in C^{\infty}(S, \mathrm{SU}(2))$. If $g$ has a factorization

$$
g(z)=k_{1}^{*}(z)\left(\begin{array}{cc}
e^{\chi(z)} & 0 \\
0 & e^{-\chi(z)}
\end{array}\right) k_{2}(z)
$$

where $\chi \in C^{\infty}(S, i \mathbb{R})$, and $k_{1}$ and $k_{2}$ are as in (I.1) and (II.1), respectively, of Theorem 1.1, then $E(g)$, the holomorphic $\mathrm{SL}(2, \mathbb{C})$ bundle on $\widehat{\Sigma}$ defined by $g$ as a transition function, is semistable, and the associated bundle for the defining representation has a sub-line bundle with an antiholomorphic reflection symmetry compatible with $R$.

\section{Remark 1.4.}

(a) For a multiloop $g$ as in the theorem (or more generally for a multiloop with minimal smoothness), we explain how $g$ defines a holomorphic bundle in Appendix A.

(b) Because of the residual symmetry condition on the sub-line bundle in the theorem, the existence of a factorization as in the theorem is not a generic condition, except in the classical case (this is explained further in Appendix A.4).

(c) As an example of our ignorance, suppose that $g: S \rightarrow \mathrm{SU}(2)$ has a factorization as in the theorem. Is the same true for the inverse, $g^{*}$ ? This is true in the classical case, because in the classical case there exists a root subgroup factorization if and only if there is a triangular factorization, and for the latter condition, $g=\operatorname{lmau}$ is a triangular factorization if and only if $g^{-1}=u^{*} m^{*} a l^{*}$ is a triangular factorization. We suspect the answer is negative in general.

A basic open question is whether a generic $g: S \rightarrow \mathrm{SU}(2)$ can be factored as a product of $\mathrm{SU}(2)$-valued multiloops

$$
g(z)=\left(\begin{array}{cc}
a^{*}(z) & -b(z) \\
b^{*}(z) & a(z)
\end{array}\right)\left(\begin{array}{cc}
e^{\chi(z)} & 0 \\
0 & e^{-\chi(z)}
\end{array}\right)\left(\begin{array}{cc}
d^{*}(z) & -c^{*}(z) \\
c(z) & d(z)
\end{array}\right)
$$

where $a, b, c, d$ are appropriate boundary values (depending on the smoothness of $g$ ) of holomorphic functions in $\Sigma$. Note that in Theorem 1.3, $a$ and $b(c$ and $d)$ are not allowed to simultaneously vanish in $\Sigma$; the point is that this condition has to be relaxed. We will discuss some results along these lines in Sections 3.1, 4.2 and 4.3.

\subsection{Spin Toeplitz operators}

Assume that $\Sigma$ has a spin structure. There is an induced spin structure for $\widehat{\Sigma}$ which has an antiholomorphic reflection symmetry compatible with $R$ (see [20, Chapter 7]). For simplicity we additionally assume that the $\bar{\partial}$ operator for spinors on $\widehat{\Sigma}$ is invertible. In this case there is a (pre-)Hilbert space polarization for the space of $\left(\mathbb{C}^{2}\right.$ valued) spinors along $S$,

$$
\Omega^{1 / 2}(S) \otimes \mathbb{C}^{2}=H^{1 / 2}(\Sigma) \otimes \mathbb{C}^{2} \oplus H^{1 / 2}\left(\Sigma^{*}\right) \otimes \mathbb{C}^{2},
$$


where $H^{1 / 2}(\Sigma)$ denotes the space of holomorphic spinors on $\Sigma$. Given a (measurable) loop $g: S \rightarrow \mathrm{SU}(2)$, there is an associated unitary multiplication operator $M_{g}$ on $\Omega^{1 / 2}(S) \otimes \mathbb{C}^{2}$, and relative to the polarization

$$
M_{g}=\left(\begin{array}{ll}
A(g) & B(g) \\
C(g) & D(g)
\end{array}\right)
$$

In the classical case $A(g)(B(g))$ is the classical block Toeplitz operator (Hankel operator, respectively), associated to the symbol $g$. In general the 'spin Toeplitz (Hankel) operators' $A(g)$ $(B(g)$, respectively) have many of the same qualitative properties as in the classical case, because the projection $\Omega^{1 / 2} \rightarrow H^{1 / 2}$ differs from the classical projection by a smoothing operator.

Theorem 1.5. Suppose that $g: S \rightarrow \mathrm{SU}(2)$ (is smooth and) has a factorization as in Theorem 1.3. Then for any choice of spin structure for which $\bar{\partial}$ is invertible,

$$
\operatorname{det}\left(A(g) A\left(g^{-1}\right)\right)=\operatorname{det}\left(A\left(k_{1}\right) A\left(k_{1}^{-1}\right)\right) \operatorname{det}\left(\dot{A}\left(e^{\chi}\right) \dot{A}\left(e^{-\chi}\right)\right)^{2} \operatorname{det}\left(A\left(k_{2}\right) A\left(k_{2}^{-1}\right)\right),
$$

where in the middle factor $\dot{A}\left(e^{\chi}\right)$ is the compression to $H^{1 / 2}(\Sigma)$ of $e^{\chi}$ as a (scalar) multiplication operator on $\Omega^{1 / 2}(S)$ (which accounts for the square of this factor).

Even in the classical case, our proof of this is far more illuminating than the one in [14]. For example we will prove the following much stronger statement, at the level of operators:

Theorem 1.6. Suppose that $k_{1}, k_{2}$ are measurable multiloops $\left.S \rightarrow \mathrm{SU}(2)\right)$ of the form

$$
k_{1}=\left(\begin{array}{cc}
a(z) & b(z) \\
-b^{*}(z) & a^{*}(z)
\end{array}\right) \quad \text { and } \quad k_{2}=\left(\begin{array}{cc}
d^{*}(z) & -c^{*}(z) \\
c(z) & d(z)
\end{array}\right)
$$

where $a, b, c$ and $d$ are boundary values of holomorphic function in $\Sigma$. Then

$$
A\left(k_{1}^{*} k_{2}\right)=A\left(k_{1}^{*}\right) A\left(k_{2}\right) .
$$

There are other senses in which the factors $k_{1}, \exp (\chi)$ and $k_{2}$ are expected to be "independent". For example in the classical case we have previously conjectured that these factors are independent random variables with respect to the large temperature limit for Wiener measure on the loop group, and that they Poisson commute with respect to the Evens-Lu homogeneous Poisson structure on LSU(2) (see [14]). To properly formulate nonclassical analogues of these conjectures, we need to prove the existence of a factorization for a generic loop, as we discussed at the end of the previous subsection.

\section{Remark 1.7.}

(a) One can replace the defining representation $\mathbb{C}^{2}$ by any representation of $\mathrm{SU}(2)$ and there is a corresponding factorization.

(b) $A(g)$ is a Fredholm operator. As elegantly explained by Quillen and Segal, $\operatorname{det}(A(g))$ can be defined as a section of a determinant line bundle, or alternatively as a function on a central extension of maps $S \rightarrow \mathrm{SU}(2)$ (see [16]). In the classical case Theorem 1.5 can be refined to yield a factorization for $\operatorname{det}(A(g))$, but this is open in general.

(c) $\operatorname{det}(A(g))$ is essentially the state corresponding to $\Sigma$ for the $\mathrm{SU}(2)$ WZW model at level one. It is interesting to ask whether there might be some factorization for the corresponding state at higher level. This is true in the classical case, but for a trivial reason: the state at level $l$ is the $l$ th power of the state at level one, i.e., one replaces the defining representation by $\left(\mathbb{C}^{2}\right)^{\otimes l}$. 


\subsection{Plan of the paper}

In Section 2 we use ideas of Krichever and Novikov to obtain a linear triangular factorization, as in (1.1), for a reasonable function $f: S \rightarrow \mathbb{C}$ (in fact for any hyperfunction). In Section 3 we prove Theorem 1.1 and a technical refinement for Holder continuous loops. In Section 3.1 we will present a generalization of Theorem 1.1 in which we relax the simultaneously nonvanishing hypotheses in (I.1) and (II.1). In Sections 4 and 5, we prove Theorems 1.3 and 1.5, respectively. In Section 4.3 we further discuss factorization and semistable bundles. In Section 5.2 we present some calculations of determinants for spin Toeplitz operators in the scalar case (the middle factor in Theorem 1.5); one suspects this can be substantially improved. Finally there is Appendix A on hyperfunctions, which we use sporadically in the text.

In part II of this paper [1], we will present a number of explicit calculations, especially for elliptic and hyperelliptic surfaces. A motivating question is whether it is possible to view spin Toeplitz operators in this paper as deformations of classical Toeplitz operators by allowing the surface to degenerate.

\section{Linear triangular factorization}

In the remainder of the paper, $\Sigma$ denotes a connected compact Riemann surface with nonempty boundary $S$. Let $\widehat{\Sigma}$ denote the double, i.e.,

$$
\widehat{\Sigma}=\Sigma^{*} \circ \Sigma,
$$

where $\Sigma^{*}$ is the adjoint of $\Sigma$, the surface $\Sigma$ with the orientation reversed, and the composition is sewing along the common boundary $S$. Let $R$ denote the antiholomorphic involution (or reflection) fixing $S$.

The singular cohomology group $H^{1}(\widehat{\Sigma}, \mathbb{R})$ is a real symplectic vector space with respect to wedge product. There is a positive polarization of the complexification

$$
H^{1}(\widehat{\Sigma}, \mathbb{C})=H^{1,0} \oplus H^{0,1},
$$

where we concretely identify $H^{1,0}$ with the complex genus $(\widehat{\Sigma})$-dimensional vector space of holomorphic differentials on $\widehat{\Sigma}$, and $H^{0,1}$ is the conjugate vector space of antiholomorphic differentials. In this way we can think of $H^{1,0}$ as the dual of $H^{0,1}$ and vice versa. There is also a Dolbeault isomorphism

$$
H^{1}(\mathcal{O}(\widehat{\Sigma})) \rightarrow H^{0,1}(\widehat{\Sigma})
$$

where $\mathcal{O}(\widehat{\Sigma})$ denotes the sheaf of holomorphic functions (see [6, p. 45], but we will spell out what we need in (2.1)).

Let $H^{0}(S)$ denotes the space of analytic functions on $S$ (hence each such function can be extended to a holomorphic function in an open neighborhood of $S$ in $\widehat{\Sigma}$ ). Using Mayer-Vietoris for the sheaf of holomorphic functions, corresponding to a slight thickening of the covering of $\widehat{\Sigma}$ by $\Sigma$ and $\Sigma^{*}$, there is an exact sequence of vector spaces

$$
0 \rightarrow H^{0}(\widehat{\Sigma}) \rightarrow H^{0}(\Sigma) \oplus H^{0}\left(\Sigma^{*}\right) \rightarrow H^{0}(S) \rightarrow H^{0,1}(\widehat{\Sigma}) \rightarrow 0 .
$$

In terms of the Dolbeault isomorphism, the connecting map $H^{0}(S) \rightarrow H^{0,1}(\widehat{\Sigma})$ is realized concretely as a map

$$
H^{0}(S) \rightarrow H^{0,1}(\widehat{\Sigma}): f \rightarrow \begin{cases}\bar{\partial} f_{1} & \text { in } \Sigma \\ \bar{\partial} f_{2} & \text { in } \Sigma^{*}\end{cases}
$$


where in the $C^{\infty}$ category, $f=f_{1}-f_{2}, f_{1}$ is smooth in (a slight open enlargement of) $\Sigma$, and $f_{2}$ is smooth in (a slight open enlargement of) $\Sigma^{*}$.

In reference to the following statements, recall that the space of complex hyperfunctions on an oriented analytic one-manifold is the dual of the space of complex analytic one forms on the one-manifold, and is denoted by $\operatorname{Hyp}(S)$; see Appendix A (and [19] for the original reference). The introduction of hyperfunctions is possibly a distraction at this point of the paper. The point is that eventually we will want to consider 'transition functions' with minimal regularity; all such functions can be viewed as hyperfunctions, and the natural domain for connecting maps, such as (2.1), is a space of hyperfunctions.

Lemma 2.1. The connecting map (2.1) is dual to the injective map

$$
C^{\omega} \Omega^{1}(S, \mathbb{C}) \leftarrow H^{1,0}(\widehat{\Sigma}) \equiv H^{1}(\widehat{\Sigma}, \mathbb{R}) \leftarrow 0
$$

given by restriction of a holomorphic differential to $S$. Consequently the connecting map extends continuously to a surjective map

$$
\operatorname{Hyp}(S) \rightarrow H^{0,1}(\widehat{\Sigma}) \equiv H^{1}(\widehat{\Sigma}, \mathbb{R}) \rightarrow 0 .
$$

Proof. We first show that the connecting map is dual to the restriction map. Suppose that $f \in H^{0}(S)$ (i.e., functions which are holomorphic in a neighborhood of $S$ ) and write $f=f_{1}-f_{2}$ as in the realization of the connecting map (2.1), $f \rightarrow \bar{\partial} f_{j}$. Suppose also that $\omega \in H^{1,0}(\widehat{\Sigma})$. To prove the formula for the transpose, we must show that

$$
\left(\bar{\partial} f_{j}, \omega\right)=\left(f,\left.\omega\right|_{S}\right) .
$$

The left-hand side equals (using $d \omega=0$ and Stokes's theorem)

$$
\int_{\Sigma} \bar{\partial} f_{1} \wedge \omega+\int_{\Sigma^{*}} \bar{\partial} f_{2} \wedge \omega=\int_{S} f_{1} \wedge \omega-\int_{S} f_{2} \wedge \omega=\int_{S} f \wedge \omega
$$

and this equals the right-hand side.

The fact that the connecting map has a continuous extension to hyperfunctions follows from the fact that it is the dual of the restriction map, which is continuous. The extension is described more explicitly in Appendix A. The basic idea (due to Sato) is that a hyperfunction $f$ on $S$ can be represented as a pair, which we heuristically write as $f=f^{\prime}+f^{\prime \prime}$, where $f^{\prime}$ is holomorphic in an annular region just outside $S$ and $f^{\prime \prime}$ is holomorphic in an annular region just inside $S$. We then compute the connecting map using three open sets, the interior of $\Sigma$, a sufficiently small annular region containing $S$, and the interior of $\Sigma^{*}$. One must check that this is independent of the choice of representation for the hyperfunction: we can also represent $f=\left(f^{\prime}+g\right)+\left(f^{\prime \prime}-g\right)$, where $g$ is holomorphic in a small annular region containing $S$.

Fix a basepoint $(0) \in \Sigma^{0}:=$ interior $(\Sigma)$, and let $(\infty) \in \Sigma^{*}$ denote the reflected basepoint. The starting point for a series by papers by Krichever and Novikov (see for example [11], which summarizes several of their papers) is the following

Lemma 2.2. There exists a unique meromorphic differential dk on $\widehat{\Sigma}$ which is holomorphic in $\widehat{\Sigma} \backslash\{(0),(\infty)\}$, has simple poles at $(0),(\infty)$ with residues \pm 1 , respectively, and satisfies $\operatorname{Re}\left(\int_{\gamma} d k\right)$ $=0$, for all closed loops in $\widehat{\Sigma} \backslash\{(0),(\infty)\}$.

Proof. Let $\kappa$ denote the canonical line bundle, and $L$ the line bundle corresponding to the divisor $(0)+(\infty)$. The holomorphic sections of the line bundle $\kappa \otimes L$ are meromorphic differentials with at most simple poles at $(0),(\infty)$. The Riemann-Roch theorem implies that

$$
\operatorname{dim}\left(H^{0}(\kappa \otimes L)\right)-\operatorname{dim}\left(H^{1}(\kappa \otimes L)\right)=\operatorname{deg}(\kappa \otimes L)-(g-1),
$$


where $g=\operatorname{genus}(\widehat{\Sigma})$. But

$$
\operatorname{dim}\left(H^{1}(\kappa \otimes L)\right)=\operatorname{dim}\left(H^{0}\left(\kappa \otimes(\kappa \otimes L)^{-1}\right)\right)=\operatorname{dim}\left(H^{0}\left(L^{-1}\right)\right)=0 .
$$

Hence the dimension of the space of meromorphic differentials with at most simple poles at $(0),(\infty)$ is $g+1$. A differential cannot have a single pole. So any meromorphic differential in this space, which is not globally holomorphic, will have simple poles at $(0)$ and $(\infty)$ with the sum of the residues necessarily equal to zero. The normalizations in the statement of the theorem uniquely determine $d k$.

In the classical case $d k=d z / z$. Following Krichever and Novikov, set $\tau=\operatorname{Re}(k)$, which is a single valued harmonic function on $\widehat{\Sigma} \backslash\{(0),(\infty)\}$, which limits to $-\infty$ at $(0)$ and $+\infty$ at $(\infty)$; this is thought of as a distinguished time parameter.

The following summarizes a "linear triangular decomposition" for functions which follows immediately from the existence of $d k$. The point is that the existence of $d k$, which induces a metric on $S$, enables us to find a complement to the sum of the two subspaces, holomorphic functions in the interior of $\Sigma$, and holomorphic functions in the interior of $\Sigma^{*}$.

Proposition 2.3. A hyperfunction $\chi: \widehat{\Sigma} \rightarrow \mathbb{C}$ can be written uniquely

$$
\chi=\chi_{-}+\chi_{0}+\chi_{+}
$$

where $\chi_{-} \in H^{0}\left(\Sigma^{0 *},(\infty) ; \mathbb{C}, 0\right), \chi_{+} \in H^{0}\left(\Sigma^{0},(0) ; \mathbb{C}, 0\right)$, and $\chi_{0} d k$ is in the span of $H^{1,0}(\widehat{\Sigma})$ and $d k$.

\section{Remark 2.4.}

(a) The zeros of $d k$ are the critical points for the time parameter $\tau$. For $d k$ the number of zeros is 2 genus $(\widehat{\Sigma})$, because there are two poles, and the degree of $\kappa$ is $2 \operatorname{genus}(\widehat{\Sigma})-2$. The zeros, are located at the singular points for the time slices of the surface.

(b) From (a) it follows that the set of $\chi_{0}$ can be characterized in the following way: $\chi_{0}$ can have at most simple poles at the simple zeros of $d k$, and $\chi_{0}(0)=\chi_{0}((\infty))$. (To explain the last condition, note that $\chi_{0} d k=c_{0} d k+$ holomorphic differential, and hence $\chi_{0}-c_{0}$ must vanish at $(0),(\infty)$.)

(c) Since the complementary functions $\chi_{0}$ can have poles at points other than the basepoints $(0)$ and $(\infty)$, they do not necessarily belong to the algebra $\mathcal{M}^{0}$ of meromorphic functions which are holomorphic in $\widehat{\Sigma} \backslash\{(0),(\infty)\}$. Consequently our choice of complementary subspace is not the same as the implicit choice made by Krichiver and Novikov (this refers to the exceptional cases (1.5) after Lemma 1 in [11]). For emphasis, note our choice does not depend on the general position hypothesis for (0) in [11].

(d) Suppose that $\Sigma$ has genus zero. In this case there is an alternative splitting, where $\chi_{0}$ is locally constant on $S$. This has the advantage that there is no dependence on the choice of basepoint. We will consider this elsewhere.

For smooth functions denote the decomposition in Proposition 2.3 by

$$
\Omega^{0}(S)=H_{+} \oplus H_{0} \oplus H_{-} .
$$

Lemma 2.5. The action of multiplication by $F \in H^{0}(\Sigma)$ maps $H_{0} \oplus H_{+}$into itself. 
Proof. First note it is obvious that multiplication by $F$ maps $H_{+}$into itself.

Suppose that $\chi_{0} \in H_{0}$. Let $\chi_{0}^{(j)}$ denote a set of functions in $H_{0}$ such that $\omega_{j}:=\chi_{0}^{(j)} d k$ is a basis for the space of holomorphic differentials on $\widehat{\Sigma}$. We must show that there exist constants $c_{1}, \ldots, c_{\text {genus }(\widehat{\Sigma})}$ such that (we write these as functions of a parameter $z$, to clarify the notation)

$$
F(z) \chi_{0}(z)-\sum_{j=1}^{\operatorname{genus}(\widehat{\Sigma})} c_{j} \chi_{0}^{(j)}(z) \in H^{0}(\Sigma),
$$

i.e., this function does not have any poles in $\Sigma$. Since the set of poles of $F \chi_{0}$ (which are all simple) is a subset of the set of zeros of $d k$, this is equivalent to showing the following. Let $\left\{z_{1}^{0}, \ldots, z_{\text {genus }(\widehat{\Sigma})}^{0}\right\}$ denote the set of zeros of $d k$ in $\Sigma$ (see (b) of Remark 2.4). Then there exist unique constants $c_{1}, \ldots, c_{\text {genus }(\widehat{\Sigma})}$ such that

$$
F(z) \chi_{0}(z)-\sum_{j=1}^{\operatorname{genus}(\widehat{\Sigma})} c_{j} \chi_{0}^{(j)}(z)
$$

does not have any poles at the points $z_{1}^{0}, \ldots, z_{\text {genus }(\widehat{\Sigma})}^{0}$.

To turn this into a system of scalar equations, for each $1 \leq i \leq \operatorname{genus}(\widehat{\Sigma})$ choose a local coordinate $z_{i}$ with $z_{i}\left(z_{i}^{0}\right)=0$. Relative to this choice of coordinates, we can then speak of residues. Our claim is then equivalent to the claim that the $\operatorname{genus}(\widehat{\Sigma}) \times \operatorname{genus}(\widehat{\Sigma}) \operatorname{matrix}_{\operatorname{res}_{i}^{j}:=}$ $\operatorname{Res}\left(\chi_{0}^{(j)}, z_{i}^{0}\right)$ is nonsingular.

In the vicinity of $z_{i}^{0}$,

$$
\chi_{0}^{(j)}=\frac{\operatorname{res}_{i}^{j}}{z_{i}}+\operatorname{holomorphic}\left(z_{i}\right), \quad d k=\left(f_{1}^{(i)} z_{i}+O\left(z_{i}^{2}\right)\right) d z_{i}
$$

where the first coefficient $f_{1}^{(i)}$ is not zero, and

$$
\chi_{0}^{(j)} d k=\left(\operatorname{res}_{i}^{j} f_{1}^{(i)}+O\left(z_{i}\right)\right) d z_{i} .
$$

We can just as well show that the matrix $\operatorname{res}_{i}^{j} f_{1}^{(i)}$ is nonsingular. In invariant terms we must show that the evaluation map

$$
H^{1,0}(\widehat{\Sigma}) \rightarrow \bigoplus_{i=1}^{\operatorname{genus}(\widehat{\Sigma})} T_{z_{i}}^{*}
$$

is an isomorphism of vector spaces. A holomorphic differential in the kernel of this map would then have 2 genus $(\widehat{\Sigma})$ zeros and no poles, which is impossible since the degree of $\kappa$ is $\operatorname{2genus}(\widehat{\Sigma})-2$.

In the classical case it is a (difficult to prove but) well-known fact that if $\chi$ is Holder continuous of order $s>0$, and $s$ is nonintegral, then $\chi_{ \pm}$are Holder continuous of order $s$. This is equivalent to the fact that the Hilbert transform $\mathcal{H}:=i P_{+}-i P_{-}: \chi \rightarrow i \chi_{+}-i \chi_{-}$, which can be expressed as a principal value integral relative to the Cauchy kernel

$$
\chi(z) \rightarrow \frac{1}{\pi} p \cdot v \cdot \int_{S^{1}} \frac{\chi(\zeta)}{\zeta-z} d \zeta
$$

is a bounded operator on $C^{s}\left(S^{1}\right)$, the Banach space of Holder continuous functions of order $s$ (the original reference in the classical case is [5, Chapter III, Section 3]; this is cited for example in $[4$, p. 60]). 
Theorem 2.6. Suppose that $s>0$ and nonintegral. In reference to Proposition 2.3, if $\chi \in C^{s}(S)$ (i.e., Holder continuous of order $s$ ), then $\chi_{ \pm} \in C^{s}(S)$.

This is equivalent to proving the continuity of an analogue of the Hilbert transform on $C^{s}(S)$. The kernel is essentially the Szego kernel, which in general is the classical Cauchy integral operator plus a smooth perturbation (see [8], and [1] for examples). Thus Theorem 2.6 follows from the classical case.

\section{Proof of Theorem 1.1}

In Theorem 1.1 it is obvious that (ii) implies (iii). This actually follows from the classical example, because we can use $\mathfrak{z}$ to pull results from the sphere back to $\widehat{\Sigma}$. It is also clear that (iii) implies (i): This follows immediately by just multiplying the factors together. In the course of completing the proof of Theorem 1.1, we will also prove the following

Theorem 3.1. Suppose that $k_{1} \in C^{s}(S, \mathrm{SU}(2))$, where $s>0$ and nonintegral. The following are equivalent:

(I.1) $k_{1}$ is of the form

$$
k_{1}(z)=\left(\begin{array}{cc}
a(z) & b(z) \\
-b^{*}(z) & a^{*}(z)
\end{array}\right), \quad z \in S
$$

where $a, b \in H^{0}(\Sigma)$ have $C^{s}$ boundary values, $a((0))>0$, and a and $b$ do not simultaneously vanish at a point in $\Sigma$.

(I.3) $k_{1}$ has a factorization of the form

$$
\left(\begin{array}{cc}
1 & 0 \\
y^{*}(z)+y_{0}(z) & 1
\end{array}\right)\left(\begin{array}{cc}
a_{1} & 0 \\
0 & a_{1}^{-1}
\end{array}\right)\left(\begin{array}{cc}
\alpha_{1}(z) & \beta_{1}(z) \\
\gamma_{1}(z) & \delta_{1}(z)
\end{array}\right)
$$

where $y \in H^{0}\left(\Sigma^{0}\right), y_{0}$ is a zero mode, $a_{1}>0$, the last factor is in $H^{0}\left(\Sigma^{0}, \mathrm{SL}(2, \mathbb{C})\right)$ and is unipotent upper triangular at the basepoint (0), and the factors have $C^{s}$ boundary values.

Similarly, the following are equivalent:

(II.1) $k_{2}$ is of the form

$$
k_{2}(z)=\left(\begin{array}{cc}
d^{*}(z) & -c^{*}(z) \\
c(z) & d(z)
\end{array}\right), \quad z \in S^{1},
$$

where $c, d \in H^{0}(\Sigma)$ have $C^{s}$ boundary values, $c((0))=0, d((0))>0$, and $c$ and $d$ do not simultaneously vanish at a point in $\Sigma$.

(II.3) $k_{2}$ has a factorization of the form

$$
\left(\begin{array}{cc}
1 & x^{*}(z)+x_{0}(z) \\
0 & 1
\end{array}\right)\left(\begin{array}{cc}
a_{2} & 0 \\
0 & a_{2}^{-1}
\end{array}\right)\left(\begin{array}{cc}
\alpha_{2}(z) & \beta_{2}(z) \\
\gamma_{2}(z) & \delta_{2}(z)
\end{array}\right)
$$

where $x \in H^{0}\left(\Sigma^{0},(0) ; \mathbb{C}, 0\right), x_{0}$ is a zero mode, $x_{0}((0))=0 a_{2}>0$, the last factor is in $H^{0}\left(\Sigma^{0}, \mathrm{SL}(2, \mathbb{C})\right)$ and is upper triangular unipotent at the basepoint $(0)$, and the factors have $C^{s}$ boundary values.

The triangular factorizations in (I.3) and (II.3) are uniquely determined. 
Remark 3.2. When $k_{2}$ is the restriction to $S$ of a function with entries in $\mathcal{M}^{0}$, the determinant condition $c^{*} c+d^{*} d=1$ can be interpreted as an equality of functions in $\mathcal{M}^{0}$. Together with $d((0))>0$, this implies that $c$ and $d$ do not simultaneously vanish. Thus the simultaneous vanishing hypotheses in (I.1) and (II.1) of Theorem 3.1 are superfluous in that case.

Proof. The two sets of conditions are proven in the same way. It is obvious that (II.3) implies (II.1). The difficult task is to show that (II.1) implies (II.3).

Consider the polarization

$$
\Omega^{0}\left(S, \mathbb{C}^{2}\right)=\mathcal{H}_{+} \otimes \mathbb{C}^{2} \oplus \mathcal{H}_{-} \otimes C^{2},
$$

where $\mathcal{H}_{+}=H_{+}+\mathbb{C}$ (functions which are holomorphic in $\Sigma$ ), and $\mathcal{H}_{-}$is the sum of $H_{-}$and the subspace of zero modes $x_{0} \in H_{0}$ such that $x_{0} d k$ is a global holomorphic differential (i.e., $x_{0}((0))=0$, as in the statement of (II.3)). We write $P_{ \pm}$for the corresponding projections. We will view $\Omega^{0}\left(S, \mathbb{C}^{2}\right)$ as a preHilbert space, by using the measure induced by the restriction of $(2 \pi i)^{-1} d k$ to $S$. (Note that for a differential $\omega$, if $\omega^{*}:=\overline{R^{*} \omega}$, then $(d k)^{*}=-d k$, as follows by checking the asymptotics at $(0)$ and $(\infty)$. Thus along $S,(2 \pi i)^{-1} d k$ is real. It is also nonvanishing; see (a) of Remark 2.4. The polarization is an orthogonal direct sum.)

Relative to this polarization, we write the unitary multiplication operator corresponding to a multiloop $g: S \rightarrow \mathrm{SU}(2)$ as

$$
M_{g}=\left(\begin{array}{ll}
A & B \\
C & D
\end{array}\right) .
$$

For a smooth loop the off diagonals (Hankel type operators) will be small and $A$ and $D$ will be Fredholm.

We must show that the multiloop $k_{2}$ has a unique factorization as in (II.3), i.e., we must solve for $a_{2}, x^{*}$, and so on, in

$$
\left(\begin{array}{cc}
d^{*} & -c^{*} \\
c & d
\end{array}\right)=\left(\begin{array}{cc}
1 & x^{*}+x_{0} \\
0 & 1
\end{array}\right)\left(\begin{array}{cc}
a_{2} & 0 \\
0 & a_{2}^{-1}
\end{array}\right)\left(\begin{array}{cc}
\alpha_{2} & \beta_{2} \\
\gamma_{2} & \delta_{2}
\end{array}\right)
$$

The second row implies

$$
(c, d)=a_{2}^{-1}\left(\gamma_{2}, \delta_{2}\right)
$$

This determines $a_{2}^{-1} \gamma_{2}$ and $a_{2}^{-1} \delta_{2}$, and (using the unipotence of the third factor in (II.3)), $a_{2}^{-1}=d((0))$.

The first row implies

$$
d^{*}=a_{2} \alpha_{2}+\left(x^{*}+x_{0}\right) c, \quad-c^{*}=a_{2} \beta_{2}+\left(x^{*}+x_{0}\right) d .
$$

The first term on the right-hand side of both of these equations belongs to $\mathcal{H}_{+}$. To solve for $x^{*}+x_{0}$, we will apply a projection to get rid of these $\mathcal{H}_{+}$terms.

Consider the operator

$$
T: \mathcal{H}_{-} \rightarrow \mathcal{H}_{-} \oplus \mathcal{H}_{-}: x_{0}+x^{*} \rightarrow\left(\left(c\left(x_{0}+x^{*}\right)\right)_{-},\left(d\left(x_{0}+x^{*}\right)\right)_{-}\right),
$$

where $(\cdot)_{-}$is the projection to $\mathcal{H}_{-}$. To show that we can uniquely solve for $x^{*}+x_{0}$, we will show $T$ is injective and that $\left(\left(d^{*}\right)_{-},\left(-c^{*}\right)_{-}\right)$is in the range of $T$.

The operator $T$ is the restriction of the Fredholm operator $D\left(k_{2}\right)^{*}=D\left(k_{2}^{*}\right)$ to the subspace $\left\{\left(x_{0}+x^{*}, 0\right)\right\}$, consequently the image of $T$ is closed. $T$ is also injective. For suppose that both $\left(\left(c\left(x_{0}+x^{*}\right)\right)_{-}\right.$and $\left(d\left(x_{0}+x^{*}\right)\right)_{-}$vanish. Then

$$
c\left(x_{0}+x^{*}\right)=g \quad \text { and } \quad d\left(x_{0}+x^{*}\right)=h,
$$


where $g, h \in \mathcal{H}_{+}$. Since $c, d$ do not simultaneously vanish in $\Sigma$, this implies that $x_{0}+x^{*} \in \mathcal{H}_{+}$. But this means that $x_{0}+x^{*}$ must vanish. Thus $T$ is injective and has a closed image.

The adjoint of $T$ is given by

$$
\begin{aligned}
& \quad T^{*}: \mathcal{H}_{-} \oplus \mathcal{H}_{-} \rightarrow \mathcal{H}_{-}:\left(f_{0}+f^{*}, g_{0}+g^{*}\right) \rightarrow\left(c^{*}\left(f_{0}+f^{*}\right)+d^{*}\left(g_{0}+g^{*}\right)\right)_{-} . \\
& \text {If }\left(f_{0}+f^{*}, g_{0}+g^{*}\right) \in \operatorname{ker}\left(T^{*}\right) \text {, then } \\
& \quad c^{*} f^{*}+d^{*} g^{*}+\left(c^{*} f_{0}+d^{*} g_{0}\right)_{-}=0 .
\end{aligned}
$$

By Lemma 2.5

$$
\left(c^{*} f_{0}+d^{*} g_{0}\right)_{-}=c^{*} f_{0}+d^{*} g_{0} .
$$

Thus

$$
c^{*}\left(f_{0}+f^{*}\right)+d^{*}\left(g_{0}+g^{*}\right)=0
$$

viewed as a meromorphic function in $\Sigma^{*}$, vanishes in the closure of $\Sigma^{*}$. Because $|c|^{2}+|d|^{2}=1$ around $S$,

$$
\left(f_{0}+f^{*}, g_{0}+g^{*}\right)=\lambda^{*}\left(d^{*},-c^{*}\right),
$$

where $\lambda^{*}$ is meromorphic in $\Sigma^{*}$ and vanishes at $(\infty)$ because $d^{*}((\infty))=d((0))>0$. Thus $\lambda$ is meromorphic in $\Sigma$ and vanishes at $(0)$.

We now claim that $\left(\left(d^{*}\right)_{-},-c^{*}\right) \in \operatorname{ker}\left(T^{*}\right)^{\perp}$. To prove this, suppose that $\left(f_{0}+f^{*}, g_{0}+g^{*}\right) \in$ $\operatorname{ker}\left(T^{*}\right)$, as in the previous paragraph. Then

$$
\int_{S}\left(\left(d^{*}\right)_{-}\left(f_{0}^{*}+f\right)+\left(-c^{*}\right)\left(g_{0}^{*}+g\right)\right) d k=\int_{S} \lambda\left(d^{*} d+c^{*} c\right) d k=\int_{S} \lambda d k .
$$

(Note that constants are orthogonal to $\mathcal{H}_{-}$, and hence we could replace $\left(d^{*}\right)_{-}$by $d^{*}$.) We claim this integral vanishes. Since $\lambda d k$ is a meromorphic differential in $\Sigma$ (by the previous paragraph), this equals the sum of residues of $\lambda d k$ in $\Sigma$. Because $f_{0}^{*} d k$ and $g_{0}^{*} d k$ are holomorphic differentials (the defining characteristic for zero modes), and $c$ and $d$ do not simultaneously vanish, the only point we need to worry about is (0) (see (3.2)). Finally the residue at (0) is zero, because $\lambda((0))=0$ (by the previous paragraph).

Because $T$ has closed image, there exists $x_{0}+x^{*} \in \mathcal{H}_{-}$such that

$$
\left(d^{*}\right)_{-}=\left(\left(x_{0}+x^{*}\right) c\right)_{-} \quad \text { and } \quad-c^{*}=\left(\left(x_{0}+x^{*}\right) d\right)_{-} .
$$

We can now solve for $a_{2} \alpha_{2}$ and $a_{2} \beta_{2}$ in (3.1). We previously noted that $a_{2}^{-1}=d((0))$. Now that we have solved for the factors, the form of the factorization immediately implies $\alpha_{2} \delta_{2}-\gamma_{2} \beta_{2}=1$ on $S$; by holomorphicity of the terms, this also holds in $\Sigma$.

Theorem 2.6 implies that the projections $P_{ \pm}$are continuous on $C^{s}(S)$. Consequently the operator $T$ will be continuous and have the same properties on the $C^{s}$ completions. Hence when $k_{2} \in C^{s}$, the factors are $C^{s}$. This completes the proofs of Theorems 1.1 and 3.1.

\subsection{A generalization of Theorem 1.1}

In the preceding proof a key step is to show that $\lambda d k$ is a holomorphic differential in $\Sigma$. The proof is not sharp, in the sense that $\lambda$ itself is holomorphic, whereas for the proof to go through, we can allow $\lambda$ to have simple poles at the zeros of $d k$. By considering (3.2), this suggests that there ought to be a generalization in which $c$ and $d$ are allowed to have simultaneous zeros to first order, within the zero set of $d k$. This in turn forces $a_{2}$ to be a function. It turns out to be natural for $a_{1}$ and $a_{2}$ to be functions, for other reasons, as we will explain in Section 4.3. 
Theorem 3.3. Suppose that $k_{1} \in C^{s}(S, \mathrm{SU}(2))$, where $s>0$ and nonintegral. The following are equivalent:

(I.1) $k_{1}$ is of the form

$$
k_{1}(z)=\left(\begin{array}{cc}
a(z) & b(z) \\
-b^{*}(z) & a^{*}(z)
\end{array}\right), \quad z \in S
$$

where $a, b \in H^{0}(\Sigma)$ have $C^{s}$ boundary values, $a((0))>0$, and a and $b$ can simultaneously vanish only to first order, and this set of common zeros is the set of poles in $\Sigma$ of a zero mode.

(I.3) $k_{1}$ has a factorization of the form

$$
\left(\begin{array}{cc}
1 & 0 \\
y^{*}(z)+y_{0}(z) & 1
\end{array}\right)\left(\begin{array}{cc}
a_{1}(z) & 0 \\
0 & a_{1}(z)^{-1}
\end{array}\right)\left(\begin{array}{cc}
\alpha_{1}(z) & \beta_{1}(z) \\
\gamma_{1}(z) & \delta_{1}(z)
\end{array}\right)
$$

where $a_{1}^{-1}$ is a nonvanishing sum of a zero mode and a holomorphic function in $\Sigma$, $a_{1}((0))>0, y \in H^{0}\left(\Sigma^{0}\right)$, the last factor is in $H^{0}\left(\Sigma^{0}, \mathrm{SL}(2, \mathbb{C})\right)$ and is unipotent upper triangular at the basepoint (0), and the factors have $C^{s}$ boundary values.

Similarly, the following are equivalent:

(II.1) $k_{2}$ is of the form

$$
k_{2}(z)=\left(\begin{array}{cc}
d^{*}(z) & -c^{*}(z) \\
c(z) & d(z)
\end{array}\right), \quad z \in S^{1}
$$

where $c, d \in H^{0}(\Sigma)$ have $C^{s}$ boundary values, $c((0))=0, d((0))>0$, and $c$ and $d$ can simultaneously vanish only to first order, and this set of common zeros is the set of poles in $\Sigma$ of a zero mode.

(II.3) $k_{2}$ has a factorization of the form

$$
\left(\begin{array}{cc}
1 & x^{*}(z)+x_{0}(z) \\
0 & 1
\end{array}\right)\left(\begin{array}{cc}
a_{2}(z) & 0 \\
0 & a_{2}(z)^{-1}
\end{array}\right)\left(\begin{array}{cc}
\alpha_{2}(z) & \beta_{2}(z) \\
\gamma_{2}(z) & \delta_{2}(z)
\end{array}\right)
$$

where $a_{2}$ is a nonvanishing sum of a zero mode and a holomorphic function in $\Sigma, a_{2}((0))>0$, $x \in H^{0}\left(\Sigma^{0},(0) ; \mathbb{C}, 0\right), x_{0}$ is a zero mode, the last factor is in $H^{0}\left(\Sigma^{0}, \mathrm{SL}(2, \mathbb{C})\right)$ and is upper triangular unipotent at the basepoint (0), and the factors have $C^{s}$ boundary values.

\section{Remark 3.4.}

(a) In contrast to Theorem 1.1, we are not asserting that the triangular factorizations in (I.3) and (II.3) are uniquely determined. In particular, as the theorem is stated, $a_{1}$ and $a_{2}$ can be multiplied by nonvanishing holomorphic functions in $\Sigma$.

(b) In the preceding theorem $a_{1}^{-1}$ and $a_{2}$ are not generally zero modes. Nonconstant zero modes always vanish at some point in $\Sigma$, and this would mean that for example $c$ or $d$ would have a pole. We want to avoid poles, because for example the results in Section 5 are dependent on the assumption that $a, b, c, d$ are holomorphic in $\Sigma$.

Proof. The two sets of conditions are proven in the same way. It is obvious that (II.3) implies (II.1). As before, the difficult task is to show that (II.1) implies (II.3).

Assuming (II.1), we must show that the multiloop $k_{2}$ has a factorization as in (II.3), i.e., we must prove existence of $a_{2}, x^{*}$, and so on, in

$$
\left(\begin{array}{cc}
d^{*} & -c^{*} \\
c & d
\end{array}\right)=\left(\begin{array}{cc}
1 & x^{*}+x_{0} \\
0 & 1
\end{array}\right)\left(\begin{array}{cc}
a_{2} & 0 \\
0 & a_{2}^{-1}
\end{array}\right)\left(\begin{array}{ll}
\alpha_{2} & \beta_{2} \\
\gamma_{2} & \delta_{2}
\end{array}\right) .
$$


The second row implies

$$
(c, d)=a_{2}^{-1}\left(\gamma_{2}, \delta_{2}\right)
$$

This determines $a_{2}^{-1} \gamma_{2}$ and $a_{2}^{-1} \delta_{2}$. In the context of the previous subsection, i.e., when $c$ and $d$ do not simultaneously vanish, $a_{2}$ is a constant, determined by the unipotence of the third factor in (II.3), which (in general) implies $a_{2}^{-1}((0))=d((0))$. In general, towards determining $a_{2}$, note that $a_{2}$ must have simple poles at the common zeros for $c$ and $d$, and cannot have zeros in $\Sigma$, so that $\gamma_{2}$ and $\delta_{2}$ are holomorphic and do not simultaneously vanish in $\Sigma$. The hypotheses of (II.1) guarantee that there is a zero mode with the appropriate (necessarily simple) poles in $\Sigma$. Fix a slight open enlargement of $\Sigma$. Since this surface is open, we can find a nonvanishing meromorphic function in this enlargement with the same singular behavior as this zero mode; the difference between this meromorphic function, which is our choice for $a_{2}$, and the given zero mode is holomorphic in $\Sigma$. As we remarked above, this choice is far from uniquely determined, and it is not clear how to pin down a preferred choice.

The first row implies

$$
d^{*}=a_{2} \alpha_{2}+\left(x^{*}+x_{0}\right) c, \quad-c^{*}=a_{2} \beta_{2}+\left(x^{*}+x_{0}\right) d .
$$

The basic complication, compared to the proof of Theorem 1.1, is that $a_{2} \alpha_{2}$ and $a_{2} \beta_{2}$ are no longer necessarily holomorphic in $\Sigma$, because $a_{2}$ has poles. We will first solve (not necessarily uniquely) for $x^{*}$, by using the same strategy as in the proof of Theorem 1.1, but using a different polarization.

Consider the polarization

$$
\Omega^{0}\left(S, \mathbb{C}^{2}\right)=\mathcal{H}_{+} \otimes C^{2} \oplus \mathcal{H}_{-} \otimes C^{2}
$$

where $\mathcal{H}_{+}$is now the sum of functions which are holomorphic in $\Sigma$ and zero modes, and $\mathcal{H}_{-}=H_{-}$. We write $P_{+}$, and $P_{-}$for the projections onto the subspaces $\mathcal{H}_{+} \otimes \mathbb{C}^{2}$ and $H_{-} \otimes C^{2}$, respectively.

Relative to this polarization, we write the multiplication operator defined by a multiloop as

$$
M_{g}=\left(\begin{array}{cc}
A & B \\
C & D
\end{array}\right)
$$

For a smooth loop the off diagonals (Hankel type operators) will be small and $A$ and $D$ will be Fredholm.

Consider the operator

$$
T: \mathcal{H}_{-} \rightarrow \mathcal{H}_{-} \oplus \mathcal{H}_{-}: x^{*} \rightarrow\left(\left(\left(c x^{*}\right)_{-},\left(d x^{*}\right)_{-}\right),\right.
$$

where $(\cdot)_{-}$is shorthand for the projection to $\mathcal{H}_{-}$. The operator $T$ is the restriction of the Fredholm operator $D\left(k_{2}\right)^{*}=D\left(k_{2}^{*}\right)$ to the subspace $\left\{\left(x^{*}, 0\right)\right\}$, consequently the image of $T$ is closed. Unfortunately in general $T$ is not injective. For suppose that both $\left(\left(c x^{*}\right)_{-}\right.$and $\left(d x^{*}\right)_{-}$ vanish. Then

$$
c x^{*}=g \quad \text { and } \quad d x^{*}=h,
$$

where $g, h \in \mathcal{H}_{+}$. At a common simple zero for $c$ and $d$, it could happen that $g, h$ also have a common pole, so $x^{*}$ does not necessarily have simple poles.

The adjoint of $T$ is given by

$$
T^{*}: \mathcal{H}_{-} \oplus \mathcal{H}_{-} \rightarrow \mathcal{H}_{-}:\left(f^{*}, g^{*}\right) \rightarrow\left(c^{*} f^{*}+d^{*} g^{*}\right)_{-} .
$$


If $\left(f^{*}, g^{*}\right) \in \operatorname{ker}\left(T^{*}\right)$, then

$$
c^{*} f^{*}+d^{*} g^{*}=0
$$

viewed as a meromorphic function in $\Sigma^{*}$. Because $|c|^{2}+|d|^{2}=1$ around $S$,

$$
\left(f^{*}, g^{*}\right)=\lambda^{*}\left(d^{*},-c^{*}\right),
$$

where $\lambda^{*}$ is meromorphic in $\Sigma^{*}$ and vanishes at $(\infty)$ because $d^{*}((\infty))=d((0))>0$. Thus $\lambda$ is meromorphic in $\Sigma$ and vanishes at (0).

We now claim that $\left(\left(d^{*}\right)_{-},-c^{*}\right) \in \operatorname{ker}\left(T^{*}\right)^{\perp}$. To prove this, suppose that $\left(f^{*}, g^{*}\right) \in \operatorname{ker}\left(T^{*}\right)$, as in the previous paragraph. Then

$$
\int_{S}\left(\left(d^{*}\right)_{-}(f)+\left(-c^{*}\right)(g)\right) d k=\int_{S} \lambda\left(d^{*} d+c^{*} c\right) d k=\int_{S} \lambda d k .
$$

We claim this integral vanishes. Since $\lambda d k$ is a meromorphic differential in $\Sigma$ (by the previous paragraph), this equals the sum of residues of $\lambda d k$ in $\Sigma$. Because the poles of $\lambda$ occur at common zeros of $c, d$, and hence only at zeros of $d k$, and because $\lambda((0))=0, \lambda d k$ is holomorphic in $\Sigma$ (by the previous paragraph). Thus the integral vanishes.

Because $T$ has closed image, there exists $x^{*} \in \mathcal{H}_{-}$such that

$$
\left(d^{*}\right)_{-}=\left(x^{*} c\right)_{-} \quad \text { and } \quad-c^{*}=\left(x^{*} d\right)_{-} .
$$

(Here and below we are using the fact that $a_{2} \in H_{0}+H_{+}, \alpha_{2} \in H_{+}$, and Lemma 2.5, to conclude that $a_{2} \alpha_{2} \in H_{0}+H_{+}$, so that it is killed by $(\cdot)_{-}$.)

Now consider the operator

$$
\begin{aligned}
\mathcal{T}: & \left(H_{+}+\mathbb{C}\right) \oplus H_{0} \oplus H_{+} \rightarrow\left(H_{+}+H_{0}\right) \oplus\left(H_{+}+H_{0}\right): \\
& \left(\alpha_{2}, x_{0}, \beta_{2}\right) \rightarrow\left(a_{2} \alpha_{2}+x_{0} c, a_{2} \beta_{2}+x_{0} d\right) .
\end{aligned}
$$

This is well-defined by Lemma 2.5 .

We must show that $\left(d^{*}-x_{0} c,-c^{*}-x_{0} d\right)$ (which is in the target of $\mathcal{T}$ by the previous paragraph) is in the image of $\mathcal{T}$. The operator $\mathcal{T}$ has a closed image. The (Hilbert space) adjoint of $\mathcal{T}$ is given by

$$
\begin{aligned}
& \left(H_{+}+H_{0}\right) \oplus\left(H_{+}+H_{0}\right) \rightarrow\left(H_{+}+\mathbb{C}\right) \oplus H_{0} \oplus H_{+}: \\
& (f, g) \rightarrow\left(\left(a_{2}^{*} f\right)_{0+},\left(a_{2}^{*} g\right)_{0+},\left(c^{*} f+d^{*} g\right)_{0}\right) .
\end{aligned}
$$

We need to show that $\left(d^{*}-x_{0} c,-c^{*}-x_{0} d\right)$ is orthogonal to the kernel of $\mathcal{T}^{*}$. Suppose that $(f, g) \in \operatorname{ker}\left(\mathcal{T}^{*}\right)$ (so the three terms in (3.3) vanish). Then

$$
\left(d^{*}-x_{0} c, f\right)+\left(-c^{*}-x_{0} d, g\right)=\text { const } \cdot \int_{S}\left(d f^{*}-c g^{*}-x_{0}^{*}\left(c^{*} f+d^{*} g\right)\right) d k .
$$

The last two terms immediately drop out. Thus, up to a constant, this equals

$$
\int_{S}\left(d f^{*}-c g^{*}\right) d k=\int_{S}\left(\left(d a_{2}^{-1}\right) a_{2} f^{*}-\left(c a_{2}^{-1}\right) a_{2} g^{*}\right) d k .
$$

The vanishing of the first two terms in (3.3) imply that $a_{2} f^{*}, a_{2} g^{*} \in H_{+}$. Thus the integral vanishes since the integrand is holomorphic in $\Sigma$.

Once we have solved for the factors, the form of the factorization immediately implies $\alpha_{2} \delta_{2}-$ $\gamma_{2} \beta_{2}=1$ on $S$; by holomorphicity of the terms, this also holds in $\Sigma$. This completes the proof. 


\section{Factorization and semistability}

\subsection{Proof of Theorem 1.3}

We recall the statement of the theorem:

Theorem 4.1. Suppose $g \in C^{\infty}(S, \mathrm{SU}(2))$. If $g$ has a factorization

$$
g(z)=k_{1}^{*}(z)\left(\begin{array}{cc}
e^{\chi(z)} & 0 \\
0 & e^{-\chi(z)}
\end{array}\right) k_{2}(z)
$$

where $\chi \in C^{\infty}(S, i \mathbb{R})$, and $k_{1}$ and $k_{2}$ are as in (I.1) and (II.1), respectively, of Theorem 1.1, then $E(g)$, the holomorphic $G$ bundle on $\widehat{\Sigma}$ defined by $g$ as a transition function, is semistable, and the associated bundle for the defining representation has a sub-line bundle with an antiholomorphic reflection symmetry compatible with $R$.

Remark 4.2. In the following proof, $a_{1}$ is a positive constant and hence $a_{1}^{*}=a_{1}$. We will write $a_{1}^{*}$ at various points in the proof because in the next subsection we will want to note that the calculations are valid more generally when $a_{1}$ is a function.

Proof. In the proof we will use the fact that, using a multiloop as a transition function, there is a bijective correspondence between the double coset space

$$
H^{0}\left(\Sigma^{0 *}, G\right) \backslash \operatorname{Hyp}(S, G) / H^{0}\left(\Sigma^{0}, G\right)
$$

and the set of isomorphism classes of holomorphic $G$ bundles on $\widehat{\Sigma}$, and that a smooth function defines a hyperfunction; see Appendix A.3.

By assumption $k_{1}$ and $k_{2}$ have 'triangular factorizations' of the following forms:

$$
k_{1}=\left(\begin{array}{cc}
1 & 0 \\
y^{*}+y_{0} & 1
\end{array}\right)\left(\begin{array}{cc}
a_{1} & 0 \\
0 & a_{1}^{-1}
\end{array}\right)\left(\begin{array}{cc}
\alpha_{1} & \beta_{1} \\
\gamma_{1} & \delta_{1}
\end{array}\right),
$$

and

$$
k_{2}=\left(\begin{array}{cc}
1 & x^{*}+x_{0} \\
0 & 1
\end{array}\right)\left(\begin{array}{cc}
a_{2} & 0 \\
0 & a_{2}^{-1}
\end{array}\right)\left(\begin{array}{cc}
\alpha_{2} & \beta_{2} \\
\gamma_{2} & \delta_{2}
\end{array}\right) .
$$

Given these 'triangular factorizations' for $k_{1}$ and $k_{2}$, we can derive a 'triangular factorization' for $g$. (Note: we have not developed a general theory of triangular factorization in the context of this paper, so we are using this term somewhat loosely.) To simplify notation, let $X=$ $a_{2}^{-2}\left(x+x_{0}^{*}\right), Y=a_{1}^{2}\left(y+y_{0}^{*}\right)$, and $a=a_{1}^{*} a_{2}$. Then

$$
\begin{aligned}
g= & \left(\begin{array}{ll}
\alpha_{1} & \beta_{1} \\
\gamma_{1} & \delta_{1}
\end{array}\right)^{*}\left(\begin{array}{cc}
1 & Y \\
0 & 1
\end{array}\right)\left(\begin{array}{cc}
a e^{\chi_{-}+\chi_{0}+\chi_{+}} & 0 \\
0 & \left(a e^{\chi_{-}+\chi_{0}+\chi_{+}}\right)^{-1}
\end{array}\right)\left(\begin{array}{cc}
1 & X^{*} \\
0 & 1
\end{array}\right)\left(\begin{array}{cc}
\alpha_{2} & \beta_{2} \\
\gamma_{2} & \delta_{2}
\end{array}\right) \\
= & \left(\begin{array}{cc}
\alpha_{1}^{*} & \gamma_{1}^{*} \\
\beta_{1}^{*} & \delta_{1}^{*}
\end{array}\right)\left(\begin{array}{cc}
e^{\chi_{-}} & 0 \\
0 & e^{-\chi_{-}}
\end{array}\right)\left(\begin{array}{cc}
1 & e^{-2 \chi_{-}} Y \\
0 & 1
\end{array}\right)\left(\begin{array}{cc}
a e^{\chi_{0}} & 0 \\
0 & \left(a e^{\chi_{0}}\right)^{-1}
\end{array}\right)\left(\begin{array}{cc}
1 & e^{2 \chi_{+}} X^{*} \\
0 & 1
\end{array}\right) \\
& \times\left(\begin{array}{cc}
e^{\chi_{+}} & 0 \\
0 & e^{-\chi_{+}}
\end{array}\right)\left(\begin{array}{ll}
\alpha_{2} \\
\gamma_{2} & \delta_{2}
\end{array}\right) .
\end{aligned}
$$

The bundle $E(g)$ defined by $g$ as a transition function depends only on the product of the middle three factors, because of the remark at the beginning of the proof. The product of the middle three factors equals

$$
\left(\begin{array}{cc}
a e^{\chi_{0}} & B \\
0 & \left(a e^{\chi_{0}}\right)^{-1}
\end{array}\right)
$$


where

$$
B=a^{-1} e^{-\chi_{0}-2 \chi-} Y+a e^{\chi_{0}+2 \chi+} X^{*} .
$$

We claim that the rank two bundle defined by this transition function is semistable. Suppose otherwise. Then there exists a sub-line bundle which has positive degree. The degree of this sub-line bundle is the negative of the degree of a transition function $S \rightarrow \operatorname{GL}(1, \mathbb{C}$ ) (see our conventions for transition functions in Remark A.1). This means that there exists a factorization of the form

$$
\left(\begin{array}{cc}
a e^{\chi_{0}} & B \\
0 & \left(a e^{\chi_{0}}\right)^{-1}
\end{array}\right)=g_{-}\left(\begin{array}{cc}
\lambda & \beta \\
0 & \lambda^{-1}
\end{array}\right) g_{+}^{-1}
$$

where $g_{ \pm}=\left(\begin{array}{ll}\alpha_{ \pm} & \beta_{ \pm} \\ \gamma_{ \pm} & \delta_{ \pm}\end{array}\right)$are holomorphic $\operatorname{SL}(2, \mathbb{C})$-valued functions in $\Sigma\left(\Sigma^{*}\right.$, respectively), and the degree of $\lambda: S \rightarrow \mathrm{GL}(1, \mathbb{C})$ is negative. This factorization implies

$$
a e^{\chi_{0}} \gamma_{-}=\lambda^{-1} \gamma_{+}
$$

and hence the line bundle defined by the transition function $\left(a e^{-\chi_{0}} \lambda\right)^{-1}$ has a global holomorphic section. But this is impossible, because the degree of this transition function is positive (i.e., the degree of the corresponding line bundle is negative). This implies that $E(g)$ is semistable. (Note that in general, a principal bundle is semistable if and only if the corresponding adjoint bundle is semistable; however for $\operatorname{SL}(2, \mathbb{C})$ it suffices to consider the defining representation, see [17].)

Because $\chi_{0}=-\chi_{0}^{*}$, and because $a$ is a positive constant, the line bundle defined by the transition function $a e^{\chi_{0}}$ has antiholomorphic symmetry with respect to $R$. This completes the proof.

\subsection{A generalization of Theorem 1.3}

Theorem 4.3. Suppose $g \in C^{\infty}(S, \mathrm{SU}(2))$. If $g$ has a factorization

$$
g(z)=k_{1}^{*}(z)\left(\begin{array}{cc}
e^{\chi(z)} & 0 \\
0 & e^{-\chi(z)}
\end{array}\right) k_{2}(z)
$$

where $\chi \in C^{\infty}(S, i \mathbb{R})$, and $k_{1}$ and $k_{2}$ are as in (I.1) and (II.1), respectively, of Theorem 3.3, then $E(g)$, the holomorphic $G$ bundle on $\widehat{\Sigma}$ defined by $g$ as a transition function, is semistable.

Proof. For $a_{1}$ and $a_{2}$ as in Theorem 3.3 (viewed as transition functions)

$$
\operatorname{deg}\left(a_{1}^{*} a_{2}\right)=\operatorname{deg}\left(a_{1}^{-1}\right)+\operatorname{deg}\left(a_{2}\right)
$$

Since $a_{1}^{-1}$ and $a_{2}$ do not vanish in $\Sigma$, this degree is the negative of the sum of the number of common zeros of $(a, b)$ in $\Sigma$ and the number of common zeros of $(c, d)$ in $\Sigma$. In particular the degree of $a=a_{1}^{*} a_{2}$ is negative. With the exception of the last paragraph, we can now repeat the preceding proof verbatim; the key point is that the degree of the transition function $\left(a e^{-\chi_{0}} \lambda\right)^{-1}$, calculated in the penultimate paragraph, remains positive.

The main point of this theorem is that the antiholomorphic symmetry has been broken. Consequently there is now a chance that the factorization of $g$, as in the theorem, is generic. 


\subsection{Do there exist converses?}

As of this writing, the questions of whether there are converses to Theorems 1.3 and 4.3 are open. We briefly discuss the issues involved. In this subsection, for simplicity, we assume that $S$ has one connected component. We first consider Theorem 1.3.

Suppose that $g \in C^{\omega}(S ; \mathrm{SU}(2, \mathbb{C}))$ and $E(g)$ is semistable. Any holomorphic vector bundle on a Riemann surface has a flag of holomorphic subbundles. In particular for $E(g)$, there exists a holomorphic sub-line bundle in the associated vector bundle for the defining representation. This implies the existence of a factorization of the form

$$
g=\left(\begin{array}{ll}
l_{11} & l_{12} \\
l_{21} & l_{22}
\end{array}\right)\left(\begin{array}{cc}
\lambda & B^{\prime} \\
0 & \lambda^{-1}
\end{array}\right)\left(\begin{array}{ll}
u_{11} & u_{12} \\
u_{21} & u_{22}
\end{array}\right)
$$

where $l \in H^{0}\left(\Sigma^{*}, \mathrm{SL}(2, \mathbb{C})\right), u \in H^{0}(\Sigma, \mathrm{SL}(2, \mathbb{C})), \lambda: S \rightarrow \mathrm{GL}(1, \mathbb{C})$ has degree zero (by semistability), and $B^{\prime}: S \rightarrow \mathbb{C}$. To proceed rigorously, we would need to know more about this kind of factorization, but in this heuristic discussion, we will put this aside. If we assume that the line bundle has an antiholomorphic symmetry compatible with $R$, then the degree of the line bundle is zero, and because we are assuming $S$ is connected, we can assume that $\lambda=a^{\prime} \exp \left(\chi_{0}\right)$, where $\chi_{0}=-\chi_{0}^{*}$ and $a^{\prime}$ is a positive constant (we eventually want $a^{\prime}=a_{1} a_{2}$ ). By comparing with the factorization of $g$ in the previous subsection, we see that (putting questions about uniqueness aside)

$$
l_{11}=\alpha_{1}^{*} \exp \left(\chi_{-}\right), \quad l_{21}=\beta_{1}^{*} \exp \left(\chi_{-}\right), \quad u_{21}=\gamma_{2} \exp \left(-\chi_{+}\right), \quad u_{22}=\delta_{2} \exp \left(-\chi_{+}\right) .
$$

Given this, one can possibly mimic the calculations in [15, Section 3] to reconstruct the factorization. A key point here is that we need to know there does exist a unitary transition function; this is discussed in Appendix A.4.

Theorem 4.3 asserts the existence of a diagram of the form,

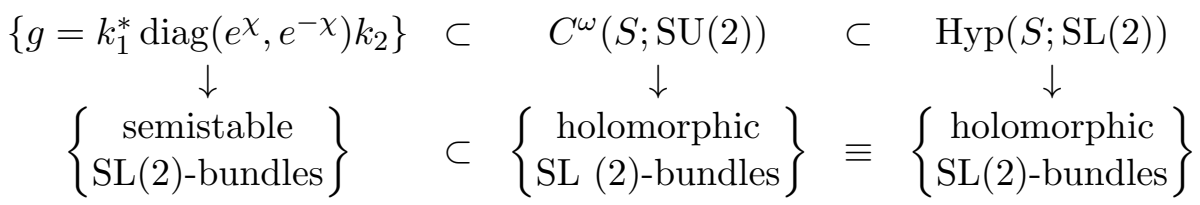

where $k_{1}$ and $k_{2}$ satisfy the conditions in Theorem 3.3, and isomorphic bundles are identified. The question is whether the first down arrow is onto (for the second down arrow, see Appendix A.4). To understand what is needed, suppose that $E(g)$ is semistable and there is a factorization as in (4.1). To get started, we need a factorization of the form $\lambda^{\prime}=a_{1}^{*} a_{2}$ (for some $\lambda^{\prime}$ equivalent to $\lambda$, as a transition function), where $a_{1}$ and $a_{2}$ are functions as in Section 3.1 and Theorem 4.3.

Remark 4.4. To develop some intuition, we need to be able to solve the following problem: Consider a decomposable bundle defined by a transition function $\operatorname{diag}\left(a, a^{-1}\right)$ where $a$ is positive function along $S$ (e.g., $a=\exp \left(\chi_{1}\right)$, where $\chi_{1}$ is a zero mode with $\chi_{1}=\chi_{1}^{*}$ ). Find a multiloop $g: S \rightarrow \mathrm{SU}(2, \mathbb{C})$ which maps to this bundle.

\section{$5 \quad$ Spin Toeplitz operators}

A spin structure for a Riemann surface is the same thing as a choice of square root for the canonical bundle, $\kappa$ (by way of explanation, holomorphic sections of $\kappa$ are holomorphic differentials). We let $\kappa^{1 / 2}\left(\rightarrow \Sigma, \widehat{\Sigma}, \Sigma^{*}\right.$, depending on the surface) denote the choice of square root (see [20, 
Chapter 7] for other points of view). There is a natural (pre-) Hilbert space structure on $\Omega^{0}\left(S, \kappa^{1 / 2}\right) \otimes \mathbb{C}^{2}\left(\mathbb{C}^{2}\right.$ valued spinors along $\left.S\right)$ given by

$$
\langle\psi, \phi\rangle=\int_{S}(\psi, \bar{\phi})_{\mathbb{C}^{2}},
$$

where $(v, \bar{w})_{\mathbb{C}^{2}}$ denotes the standard Hermitian inner product on $\mathbb{C}^{2}$, so that the integrand is a one density on $S$.

For the sheaf of holomorphic sections of $\kappa^{1 / 2}$, and (a slight open thickening of) the covering of $\widehat{\Sigma}$ by $\Sigma$ and $\Sigma^{*}$, there is a Mayer-Vietoris long exact sequence,

$$
\begin{aligned}
0 & \rightarrow H^{0}\left(\widehat{\Sigma}, \kappa^{1 / 2}\right) \rightarrow H^{0}\left(\Sigma, \kappa^{1 / 2}\right) \oplus H^{0}\left(\Sigma^{*}, \kappa^{1 / 2}\right) \\
& \rightarrow \Omega^{0}\left(S, \kappa^{1 / 2}\right) \rightarrow H^{1}\left(\widehat{\Sigma}, \kappa^{1 / 2}\right) \rightarrow 0 .
\end{aligned}
$$

In terms of the $\bar{\partial}$ operator, $H^{0}\left(\widehat{\Sigma}, \kappa^{1 / 2}\right)=\operatorname{ker}(\bar{\partial})$ and $H^{1}\left(\widehat{\Sigma}, \kappa^{1 / 2}\right)=\operatorname{coker}(\bar{\partial})$. The index of $\bar{\partial}$ is zero.

When $\bar{\partial}$ is invertible, a generic condition, by taking Hilbert space completions, we obtain a Hilbert space polarization

$$
L^{2} \Omega^{1 / 2}(S) \otimes \mathbb{C}^{2}=H_{+} \oplus H_{-},
$$

where $L^{2} \Omega^{1 / 2}(S), H_{+}$, and $H_{-}$denote the completions of $\Omega^{0}\left(S, \kappa^{1 / 2}\right), H^{0}\left(\Sigma, \kappa^{1 / 2}\right) \otimes \mathbb{C}^{2}$, and $H^{0}\left(\Sigma^{*}, \kappa^{1 / 2}\right) \otimes \mathbb{C}^{2}$, respectively. When $\bar{\partial}$ is not invertible, there are two distinct reasonable polarizations. The first possibility is that $H_{+}$is the completion of $\Omega^{0}\left(S, \kappa^{1 / 2}\right) \otimes \mathbb{C}^{2}$, and $H_{-}$is the orthogonal complement. The second possibility is that $H_{-}$is the completion of $H^{0}\left(\Sigma^{*}, \kappa^{1 / 2}\right) \otimes \mathbb{C}^{2}$ and $H_{+}$is the orthogonal complement.

Given an essentially bounded matrix valued function $g: S \rightarrow \mathcal{L}\left(\mathbb{C}^{2}\right)$, there is an associated bounded multiplication operator $M_{g}$ on $\Omega^{1 / 2}(S) \otimes \mathbb{C}^{2}$, and relative to a polarization as in the preceding paragraphs

$$
M_{g}=\left(\begin{array}{ll}
A(g) & B(g) \\
C(g) & D(g)
\end{array}\right)
$$

If $\Sigma=D$, the unit disk, then $A(g)$ and $B(g)$ are the classical (block) Toeplitz and Hankel operators associated to the symbol $g$ (see [3] and [12, Chapter 3]). In general the basic qualitative properties of the operators $A(g)$ and $B(g)$ (which we refer to as 'spin Toeplitz and Hankel operators', respectively) are the same as in the classical case, because the projection to $H_{+}$ differs from the classical projection by a smoothing operator. For example (of most importance for our purposes), exactly as in [16, Chapter 5], the map $g \rightarrow M_{g}$ defines an embedding of loops into the Hilbert-Schmidt general linear group; the precise statement is that there is an embedding

$$
L^{\infty} \cap W^{1 / 2}(S ; \mathrm{SL}(2, \mathbb{C})) \rightarrow \mathrm{GL}\left(H_{+} \oplus H_{-}\right)_{(2)} .
$$

The relevant Lie structure is described in [16, Chapters 6 and 7].

Remark 5.1. The Krichever-Novikov theory, at least in principle, provides an explicit means to calculate spin Hankel and Toeplitz operators. According to Krichever and Novikov (following the notation in [11]), for certain spin structures and for generic basepoints, there is an (orthonormal) basis for $\mathcal{M}^{1 / 2}$ (meromorphic spinors which are regular in the complement of the basepoints) of the form

$$
\ldots, \phi_{3 / 2}, \phi_{1 / 2}, \phi_{-1 / 2} \phi_{-3 / 2}, \ldots
$$


(which in the classical case reduces to the usual basis

$$
\left.\ldots, z^{2}(d z)^{1 / 2}, z(d z)^{1 / 2}, z^{0}(d z)^{1 / 2}, z^{-1}(d z)^{1 / 2}, \ldots\right)
$$

a basis for $\mathcal{M}^{0},\left\{f_{k}: k \in \frac{g}{2}+\mathbb{Z}\right\}$ (which in the classical case reduces to the usual basis), and constants $Q$ such that

$$
f_{n} \phi_{m}=\sum_{|k| \leq g / 2} Q_{n, m}^{k} \phi_{n+m-k} .
$$

Thus for each $n$, the matrices for the multiplication operator, and the spin Toeplitz operator, defined by $f_{n}$ will have band width (the number of nonzero diagonals that appear) equal to $g+1, g=\operatorname{genus}(\hat{\Sigma})$ (in the classical case $f_{n}$ is a shift operator, and the bandwidth is one). We will pursue this in part II of this paper [1].

\subsection{Determinants: proof of Theorem 1.5}

Theorem 5.2. Suppose that $g$ has a factorization as in Theorem 1.3. Then for any choice of spin structure and polarization as in the preceding subsection,

$$
\operatorname{det}\left(A(g) A\left(g^{-1}\right)\right)=\operatorname{det}\left(A\left(k_{1}\right) A\left(k_{1}^{-1}\right)\right) \operatorname{det}\left(A\left(e^{\chi}\right) A\left(e^{-\chi}\right)\right) \operatorname{det}\left(A\left(k_{2}\right) A\left(k_{2}^{-1}\right)\right) .
$$

This theorem will follow from Theorem 5.3 and the lemmas below. To place the calculations in some context, recall (see [16, pp. 88-89]) that for the universal central extension (for the Hilbert-Schmidt general linear group)

$$
0 \rightarrow \mathbb{C}^{*} \rightarrow \widetilde{\mathrm{GL}}\left(H_{+} \oplus H_{-}\right)_{(2)} \rightarrow \mathrm{GL}\left(H_{+} \oplus H_{-}\right)_{(2)}
$$

and the local cross-section (defined on the open dense set of $g$ such that $A(g)$ is invertible)

$$
g \rightarrow[g, A(g)]
$$

the multiplication is given by

$$
\tilde{g}_{1} \tilde{g}_{2}=\mathbf{c}\left(g_{1}, g_{2}\right) \tilde{g}_{3},
$$

where the cocycle is given by

$$
\begin{aligned}
\mathbf{c}(g, h)^{-1} & =\operatorname{det}\left(A(g h) A(h)^{-1} A(g)^{-1}\right)=\operatorname{det}\left(A(g)^{-1} A(g h) A(h)^{-1}\right) \\
& =\operatorname{det}\left(1+A(g)^{-1} B(g) C(h) A(h)^{-1}\right) .
\end{aligned}
$$

The corresponding Lie algebra cocycle is given by

$$
\begin{aligned}
& \omega: \mathfrak{g l}\left(H_{+} \oplus H_{-}\right)_{(2)} \times \mathfrak{g l}\left(H_{+} \oplus H_{-}\right)_{(2)} \rightarrow \mathbb{C}, \\
& \omega(X, Y)=\operatorname{tr}([A(X), A(Y)]-A([X, Y]))=\operatorname{tr}(B(Y) C(X)-B(X) C(Y)) .
\end{aligned}
$$

We are interested in the restriction of the cocycle to $\Omega^{0}(S, \operatorname{SL}(2, \mathbb{C}))$ via the injection (5.1). The basic fact about this cocycle, which we will use repeatedly, is that if $g_{-} \in H^{0}\left(\Sigma^{*}, \operatorname{SL}(2, \mathbb{C})\right)$ and $g_{+} \in H^{0}(\Sigma, \mathrm{SL}(2, \mathbb{C}))$, then

$$
\mathbf{c}\left(g_{-} g, h g_{+}\right)=\mathbf{c}(g, h) \text {. }
$$

This follows from $A\left(g_{-} g\right)=A\left(g_{-}\right) A(g)$ and $A\left(h g_{+}\right)=A(h) A\left(g_{+}\right)$, which in turn follow from $B\left(g_{-}\right)=0$ and $C\left(g_{+}\right)=0$, respectively. To see this, suppose that $H_{+}$is the completion of 
$\Omega^{0}\left(\Sigma, \kappa^{1 / 2}\right) \otimes \mathbb{C}^{2}$. Given a spinor $\phi_{+} \in H_{+}$, the product $g_{+} \phi_{+}$is holomorphic in $\Sigma$, hence $g_{+} \phi_{+} \in H_{+}$. This implies $C\left(g_{+}\right)=0$. Now consider a spinor $\phi \in H_{-}$, i.e., $\phi$ is orthogonal to $H_{+}$. We claim that $g_{-} \phi$ is also orthogonal to $H_{+}$. If $\psi_{+} \in H_{+}$, then the pointwise inner product (along $S$ )

$$
\left(\psi, \overline{g_{-} \phi}\right)_{\mathbb{C}^{2}}=\left(g_{-}^{*} \psi_{+}, \bar{\phi}\right)_{\mathbb{C}^{2}}
$$

Since $g_{-}^{*} \in H^{0}(\Sigma, \mathrm{SL}(2, \mathbb{C}))$, it follows that $\left\langle\psi_{+}, g_{-} \phi\right\rangle=\left\langle g_{-}^{*} \psi_{+}, \phi\right\rangle=0$. It follows that $B\left(g_{-}\right)=0$. A similar argument applies if $H_{+}$is the orthogonal complement of $\Omega^{0}\left(\Sigma^{*}, \kappa^{1 / 2}\right) \otimes \mathbb{C}^{2}$.

To prove the theorem we need to show that $\mathbf{c}(g, h)=1$ when $(g, h)=\left(k_{1}^{*}, k_{2}\right)$ and so on. In fact in some cases we can prove much stronger statements.

Theorem 5.3. $A\left(k_{1}^{*} k_{2}\right)=A\left(k_{1}^{*}\right) A\left(k_{2}\right)$. In particular $\mathbf{c}\left(k_{1}^{*}, k_{2}\right)=1$.

In the process of proving the first statement, we will prove Theorem 1.6, i.e., we will only assume that $k_{1}$ and $k_{2}$ are measureable maps $S \rightarrow \mathrm{SU}(2)$ of the appropriate form. We need to assume the loops are $W^{1 / 2}$ for the second statement to be valid.

Proof. Because

$$
A\left(k_{1}^{*} k_{2}\right)=A\left(k_{1}^{*}\right) A\left(k_{2}\right)+B\left(k_{1}^{*}\right) C\left(k_{2}\right)
$$

this equivalent to showing that $B\left(k_{1}^{*}\right) C\left(k_{2}\right)=0$. We will prove this by direct calculation. Suppose that $f:=\left(\begin{array}{l}f_{1} \\ f_{2}\end{array}\right) \in H_{+}=H^{1 / 2}(\Delta)$. Then

$$
\begin{aligned}
B\left(k_{1}^{*}\right) C\left(k_{2}\right) f & =P_{+}\left(k_{1}^{*} P_{-}\left(k_{2} f\right)\right)=P_{+}\left(k_{1}^{*}\left(\begin{array}{c}
P_{-}\left(d^{*} f_{1}-c^{*} f_{2}\right) \\
0
\end{array}\right)\right) \\
& =P_{+}\left(\left(\begin{array}{c}
a^{*} P_{-}\left(d^{*} f_{1}-c^{*} f_{2}\right) \\
0
\end{array}\right)\right)=0 .
\end{aligned}
$$

Thus $B\left(k_{1}^{*}\right) C\left(k_{2}\right)=0$. By relation (5.2) (for sufficiently smooth loops) this implies that $\mathbf{c}\left(k_{1}^{*}, k_{2}\right)=1$.

Lemma 5.4. $\mathbf{c}\left(k_{1}^{*}, \operatorname{diag}(\exp (\chi), \exp (-\chi))\right)=\mathbf{c}\left(\operatorname{diag}(\exp (\chi), \exp (-\chi)), k_{2}\right)=1$.

Proof. There exists a generalized triangular factorization

$$
\left(\begin{array}{cc}
a^{*} & -b \\
b^{*} & a
\end{array}\right)=\left(\begin{array}{cc}
\alpha_{1}^{*} & \gamma_{1}^{*} \\
\beta_{1}^{*} & \delta_{1}^{*}
\end{array}\right)\left(\begin{array}{cc}
a_{1} & 0 \\
0 & a_{1}^{-1}
\end{array}\right)\left(\begin{array}{cc}
1 & y+y_{0} \\
0 & 1
\end{array}\right)
$$

Consequently, using (5.4),

$$
\begin{aligned}
\mathbf{c}\left(\left(\begin{array}{cc}
a^{*} & -b \\
b^{*} & a
\end{array}\right),\left(\begin{array}{cc}
\exp (\chi) & 0 \\
0 & \exp (-\chi)
\end{array}\right)\right) & =\mathbf{c}\left(\left(\begin{array}{cc}
1 & y+y_{0} \\
0 & 1
\end{array}\right),\left(\begin{array}{cc}
e^{\chi} & 0 \\
0 & e^{-\chi}
\end{array}\right)\right) \\
& =\mathbf{c}\left(\exp \left(\left(\begin{array}{cc}
0 & y+y_{0} \\
0 & 0
\end{array}\right)\right), \exp \left(\left(\begin{array}{cc}
\chi & 0 \\
0 & -\chi
\end{array}\right)\right)\right) .
\end{aligned}
$$

In the last line we are considering the cocycle pairing of an element of $\Omega^{0}\left(S, N^{+}\right)$, where $N^{+}$ is the group of unipotent upper triangular matrices in $\mathrm{SL}(2, \mathbb{C})$, and an element of $\Omega^{0}(S, H)$, where $H$ is the diagonal subgroup of $\operatorname{SL}(2, \mathbb{C})$. We claim that the corresponding Lie algebra cocycle pairing for $\Omega^{0}\left(S, \mathfrak{n}^{+}\right)$and $\Omega^{0}(S, \mathfrak{h})$ is zero. To check this suppose that $X:=\left(\begin{array}{ll}0 & \beta \\ 0 & 0\end{array}\right) \in$ 
$\Omega^{0}\left(S, \mathfrak{n}^{+}\right)$and $Y:=\operatorname{diag}(\chi,-\chi) \in \Omega^{0}(S, \mathfrak{h})$. We must calculate (5.3) for these two multiloops. Then

$$
B(X) C(Y)\left(\left(\begin{array}{l}
f_{1} \\
f_{2}
\end{array}\right)\right)=\left(\begin{array}{c}
-\left(Y\left(\chi f_{2}\right)_{-}\right)_{+} \\
0
\end{array}\right) .
$$

From this it is clear that $\operatorname{tr}(B(X) C(Y))=0$. Similarly $\operatorname{tr}(B(Y) C(X))=0$. Thus the cocycle pairing for $\Omega^{0}\left(S, \mathfrak{n}^{+}\right)$and $\Omega^{0}(S, \mathfrak{h})$ is zero. It follows that the cocycle pairing for $\Omega^{0}\left(S, N^{+}\right)$and $\Omega^{0}(S, H)_{0}$ (the identity component) is trivial. This implies that

$$
\mathbf{c}\left(k_{1}^{*}, \operatorname{diag}(\exp (\chi), \exp (-\chi))\right)=1 .
$$

By the same argument $\mathbf{c}\left(\operatorname{diag}(\exp (\chi), \exp (-\chi)), k_{2}\right)=1$.

Lemma 5.5. $\mathbf{c}\left(k_{1}^{*} \operatorname{diag}(\exp (\chi), \exp (-\chi)), k_{2}\right)=\mathbf{c}\left(k_{1}^{*}, \operatorname{diag}(\exp (\chi), \exp (-\chi)) k_{2}\right)=1$.

Proof. Using (5.4)

$$
\mathbf{c}\left(k_{1}^{*} \operatorname{diag}(\exp (\chi), \exp (-\chi)), k_{2}\right)=\mathbf{c}\left(\left(\begin{array}{cc}
e^{\chi} & y+y_{0} \\
0 & e^{-\chi}
\end{array}\right),\left(\begin{array}{cc}
1 & x^{*}+x_{0} \\
0 & 1
\end{array}\right)\right) .
$$

As in the proof of the preceding lemma, this is a cocycle pairing between elements from two (connected) groups. The corresponding pairing of Lie algebra elements is trivial. So this cocycle pairing is trivial.

These lemmas imply Theorem 5.2.

\subsection{Calculating the determinant: scalar case}

Our goal is to calculate the second determinant appearing on the right-hand side of the statement in Theorem 5.2. Recall that in that statement we think of $e^{\chi}$ as a multiplication operator on scalar valued functions. We first consider the simplest possibility, which can be handled in the same way as done by Widom in the classical case (see [21]).

Proposition 5.6. Suppose that $\chi=\chi_{+}+\chi_{-}$, where $\chi_{+}$is holomorphic in $\Sigma$ and $\chi_{-}$is holomorphic in $\Sigma^{*}$. Then

(a) $\operatorname{det}\left(A\left(e^{\chi}\right) A\left(e^{-\chi}\right)\right)=\exp \left(\operatorname{tr}\left(B\left(\chi_{+}\right) C\left(\chi_{-}\right)\right)\right)$,

(b) if $\chi$ is $i \mathbb{R}$ valued, then this equals

$$
\exp \left(-\operatorname{tr}\left(B\left(\chi_{+}\right) B\left(\chi_{+}\right)^{*}\right)\right)=\exp \left(-\left|B\left(\chi_{+}\right)\right|_{\mathcal{L}_{2}}^{2}\right) .
$$

In the classical case $\Sigma=D$,

$$
\operatorname{tr}\left(B\left(\chi_{+}\right) B\left(\chi_{+}\right)^{*}\right)=\sum_{n=1}^{\infty} n\left|\chi_{n}\right|^{2} .
$$

Proof. Because $A\left(e^{\chi}\right)=A\left(e^{\chi-}\right) A\left(e^{\chi+}\right), \operatorname{det}\left(A\left(e^{\chi}\right) A\left(e^{-\chi}\right)\right)$ equals

$$
\operatorname{det}\left(A\left(e^{\chi_{-}}\right) A\left(e^{\chi_{+}}\right) A\left(e^{-\chi_{-}}\right) A\left(e^{-\chi_{+}}\right)\right)=\operatorname{det}\left(e^{A\left(\chi_{-}\right)} e^{A\left(\chi_{+}\right)} e^{A\left(-\chi_{-}\right)} e^{A\left(-\chi_{+}\right)}\right) .
$$

The Helton-Howe formula (see [10]) implies that this equals $\exp \left(\operatorname{tr}\left(\left[A\left(\chi_{-}\right), A\left(\chi_{+}\right)\right]\right)\right)$. Now observe that

$$
\begin{aligned}
{\left[A\left(\chi_{-}\right), A\left(\chi_{+}\right)\right] } & =A\left(\chi_{-}\right) A\left(\chi_{+}\right)-A\left(\chi_{+}\right) A\left(\chi_{-}\right) \\
& =A\left(\chi_{-}\right) A\left(\chi_{+}\right)-\left(A\left(\chi_{+} \chi_{-}\right)-B\left(\chi_{+}\right) C\left(\chi_{-}\right)\right)=B\left(\chi_{+}\right) C\left(\chi_{-}\right) .
\end{aligned}
$$

This implies part (a). 
For part (b) the only thing we need to comment on is the last equality. This follows from writing out the matrix for $B$, relative to the standard basis. Up to a multiple, it also follows abstractly by the $\mathrm{SU}(1,1)$ symmetry of $D$ and Schur's lemma.

Remark 5.7. It is obvious that the formula in (a) depends on the complex structure of $\Sigma$. Does it depend on the spin structure?

The shortcoming of the preceding proposition is that when $\operatorname{genus}(\widehat{\Sigma})>0$, we have not accounted for zero modes.

\section{Proposition 5.8.}

$$
\operatorname{det}\left(A\left(e^{\chi}\right) A\left(e^{-\chi}\right)\right)=\mathbf{c}\left(e^{\chi_{-}}, e^{\chi_{0}}\right) \mathbf{c}\left(e^{\chi_{0}}, e^{\chi_{+}}\right) \operatorname{det}\left(\left\{e^{-A\left(\chi_{+}\right)}, e^{A\left(\chi_{-}\right)}\right\}\right) \operatorname{det}\left(A\left(e^{\chi_{0}}\right) A\left(e^{-\chi_{0}}\right)\right) .
$$

In the case that $\chi$ has values in $i \mathbb{R}$, this equals

$$
\operatorname{det}\left(\left|\left\{A\left(e^{-\chi_{0}}\right), e^{-A\left(\chi_{+}\right)}\right\}\right|^{2}\right) \operatorname{det}\left(A\left(e^{\chi_{0}}\right) A\left(e^{\chi_{0}}\right)^{*}\right) \exp \left(-\operatorname{tr}\left(B\left(\chi_{+}\right) B\left(\chi_{+}\right)^{*}\right)\right),
$$

where $\{A, B\}=A B A^{-1} B^{-1}$ denotes the group commutator.

\section{Proof.}

$$
A\left(e^{\chi}\right) A\left(e^{-\chi}\right)=e^{A\left(\chi_{-}\right)} A\left(e^{\chi_{0}}\right) e^{A\left(\chi_{+}\right)} e^{-A\left(\chi_{-}\right)} A\left(e^{-\chi_{0}}\right) e^{-A\left(\chi_{+}\right)} .
$$

The right-hand side is of the form $x y z x^{-1} Y z^{-1}$. The conjugate of this by $z^{-1}$ equals

$$
z^{-1} x y z x^{-1} Y=\left\{z^{-1} x, y\right\} y\left\{z^{-1}, x\right\} y^{-1} y Y,
$$

where we have temporarily assumed that $y$ is invertible. This implies

$$
\begin{aligned}
\operatorname{det}\left(A\left(e^{\chi}\right) A\left(e^{-\chi}\right)\right)= & \operatorname{det}\left(\left\{e^{-A\left(\chi_{+}\right)} e^{A\left(\chi_{-}\right)}, A\left(e^{\chi_{0}}\right)\right\}\right) \\
& \times \operatorname{det}\left(\left\{e^{-A\left(\chi_{+}\right)}, e^{A\left(\chi_{-}\right)}\right\}\right) \operatorname{det}\left(A\left(e^{\chi_{0}}\right) A\left(e^{-\chi_{0}}\right)\right)
\end{aligned}
$$

(each of these determinants is the determinant of an operator of the form identity + trace class). Using the Helton-Howe formula, this equals

$$
\operatorname{det}\left(\left\{e^{-A\left(\chi_{+}\right)} e^{A\left(\chi_{-}\right)}, A\left(e^{\chi_{0}}\right)\right\}\right) \exp \left(-\operatorname{tr}\left(B\left(\chi_{+}\right) C\left(\chi_{-}\right)\right)\right) \operatorname{det}\left(A\left(e^{\chi_{0}}\right) A\left(e^{-\chi_{0}}\right)\right) .
$$

Now we use the identity

$$
\left\{z^{-1} x, y\right\}=z^{-1} x y x^{-1} y^{-1} z z^{-1} y z y^{-1} .
$$

This implies that the first determinant in the expression above satisfies

$$
\operatorname{det}\left(\left\{e^{-A\left(\chi_{+}\right)} e^{A\left(\chi_{-}\right)}, A\left(e^{\chi_{0}}\right)\right\}\right)=\operatorname{det}\left(\left\{e^{A\left(\chi_{-}\right)}, A\left(e^{\chi_{0}}\right)\right\}\right) \operatorname{det}\left(\left\{e^{A\left(\chi_{+}\right)}, A\left(e^{\chi_{0}}\right)\right\}\right) .
$$

In terms of the group cocycle notation, this equals

$$
\mathbf{c}\left(e^{\chi_{-}}, e^{\chi_{0}}\right) \mathbf{c}\left(e^{\chi_{0}}, e^{\chi+}\right) \text {. }
$$

This completes the proof.

Remark 5.9. In the classical case this determinant is nonvanishing. It would seem unlikely that this is true in general. But the expression we have produced does not seem to help in deciding this question. It should be possible to calculate the determinant for $\exp \left(\chi_{0}\right)$ exactly. 


\section{A Hyperfunctions and holomorphic bundles}

\section{A.1 Hyperfunctions on a circle}

Suppose that $X$ is an oriented analytic compact $d$-manifold. Let $C^{\omega} \Omega^{d}(X)$ denote the vector space of real analytic forms of order $d$ on $X$. There is a standard topology on this space. By definition the space of (real) hyperfunctions on $X$, denoted $\operatorname{Hyp}(X ; \mathbb{R})$, is the dual of this topological vector space; the space of complex hyperfunctions is the complexification, denoted $\operatorname{Hyp}(X ; \mathbb{C})$, or more simply $\operatorname{Hyp}(X)$ (see [9, p. 35]). In the case of $X=S^{1}$, any complex hyperfunction has a unique representation of the form

$$
f=f_{-}+f_{0}+f_{+},
$$

where $f_{-} \in H^{0}\left(\Delta^{*},(\infty) ; \mathbb{C}, 0\right), f_{0} \in \mathbb{C}$, and $f_{+} \in H^{0}(\Delta, 0 ; \mathbb{C}, 0)$ (this is how hyperfunctions were first introduced, see [19]). The corresponding functional is given by

$$
C^{\omega} \Omega^{1}\left(S^{1}\right) \rightarrow \mathbb{C}: \omega \rightarrow \int_{|z|=1-\epsilon}\left(f_{0}+f_{+}\right) \omega+\int_{|z|=1+\epsilon} f_{-} \omega
$$

where $\omega=g d z$ and $g$ is complex analytic in a collar of $S^{1}$, for sufficiently small $\epsilon$.

An integrable function $f: S^{1} \rightarrow \mathbb{C}$ naturally defines a hyperfunction by integrating an analytic form against $f$ around $S^{1}$. Note that for the linear triangular decomposition for the Fourier series of $f$, where $f_{ \pm}=\sum_{ \pm n>0} f_{n} z^{n}, f_{ \pm}$are not necessarily integrable on the circle, but they do define holomorphic functions in $\Delta$ ( $\Delta^{*}$, respectively).

\section{A.2 Nonabelian hyperfunctions on a circle}

Suppose that $G$ is a simply connected complex Lie group, e.g., $G=\operatorname{SL}(2, \mathbb{C})$.

In [16] it is observed that the group of analytic loops, $C^{\omega}\left(S^{1} ; G\right)$, is a complex Lie group. A neighborhood of the identity consists of those loops which have a unique Riemann-Hilbert factorization

$$
g=g_{-} \cdot g_{0} \cdot g_{+},
$$

where $g_{-} \in H^{0}\left(D^{*},(\infty) ; G, 1\right), g_{0} \in G, g_{+} \in H^{0}(D, 0 ; G, 1)$. A model for this neighborhood is

$$
H^{1}\left(D^{*}, \mathfrak{g}\right) \times G \times H^{1}(D, \mathfrak{g}),
$$

where the linear coordinates are determined by $\theta_{+}=g_{+}^{-1}\left(\partial g_{+}\right), \theta_{-}=\left(\partial g_{-}\right) g_{-}^{-1}$. The (left or right) translates of this neighborhood by elements of $L_{\text {fin }} K$ cover $H^{0}\left(S^{1}, G\right)$; a key point is that the transition functions are functions of a finite number of variables, in an appropriate sense (see [13, Chapter 2, Part III]).

The hyperfunction completion, $\operatorname{Hyp}\left(S^{1}, G\right)$, is modeled on the space

$$
H^{1}\left(\Delta^{*}, \mathfrak{g}\right) \times G \times H^{1}(\Delta, \mathfrak{g})
$$

and the transition functions are obtained by continuously extending the transition functions for the analytic loop space of the preceding paragraph. The global definition is

$$
\operatorname{Hyp}\left(S^{1}, G\right)=\lim _{r \downarrow 1} H^{0}(\{1<|z|<r\}, G) \times_{H^{0}\left(S^{1}, G\right)} \lim _{r \uparrow 1} H^{0}(\{r<|z|<1\}, G) \text {. }
$$

From this point of view, a hyperfunction is an equivalence class $[g, h]$, where $g(h)$ is a $G$-valued holomorphic map in an annulus to the left (right, respectively) of $S^{1}$ ). The correct way to 
topologize this space does not seem clear from this point of view, and we will put this aside. From the global definition it is clear that the group $H^{0}\left(S^{1}, G\right)$ acts naturally from both the left and right of $\operatorname{Hyp}\left(S^{1}, G\right)$, e.g., for the left action, $g_{l}:[g, h] \rightarrow\left[g_{l} g, h\right]$. There is a generalized Birkhoff decomposition

$$
\operatorname{Hyp}\left(S^{1}, G\right)=\bigsqcup_{\lambda \in \operatorname{Hom}\left(S^{1}, T\right)} \Sigma_{\lambda}^{\text {hyp }}, \quad \Sigma_{\lambda}^{\text {hyp }}=H^{0}\left(\Delta^{*}, G\right) \cdot \lambda \cdot H^{0}(\Delta, G) .
$$

The top stratum (the piece with $\lambda=1$ above) is open and dense, and for each point $g$ in the top stratum, there is a unique factorization as in (A.1), where $g_{ \pm}$are $G$-valued holomorphic functions in the open disks $\Delta$ and $\Delta^{*}$, respectively. We will refer to $g_{-}, g_{0}, g_{+}\left(\theta_{-}, g_{0}, \theta_{+}\right.$, respectively) as the Riemann-Hilbert coordinates (linear Riemann-Hilbert coordinates, respectively) of $g$ (see [13, Chapter 2, Part III]).

Given a continuous function $g: S^{1} \rightarrow G$ there exists a generalized Riemann-Hilbert factorization, where the factors $g_{ \pm}$are not necessarily continuous (see [4, Theorem 1.1 of Chapter VIII] for a precise statement). In this way any reasonable loop in $G$ can be regarded as an element of $\operatorname{Hyp}\left(S^{1}, G\right)$.

The space $\operatorname{Hyp}\left(S^{1}, G\right)$ depends only on the orientation and real analytic structure of $S^{1}$, i.e., there is a natural action of real analytic homeomorphisms on hyperfunctions: in terms of the global definition (A.2)

$$
\sigma:[g, h] \rightarrow\left[\sigma^{-*} g, \sigma^{-*} h\right] .
$$

Consequently if $S$ is an oriented real analytic one-manifold, then $\operatorname{Hyp}(S, G)$ is well-defined.

\section{A.3 Hyperfunctions and holomorphic $G$ bundles}

Suppose that $\widehat{\Sigma}$ is a closed Riemann surface. In this subsection it is not necessary to assume that $\widehat{\Sigma}$ is a double. We also suppose that $c: S^{1} \rightarrow \widehat{\Sigma}$ is a real analytic embedding, i.e., a simple analytic loop. We could more generally suppose that $c$ is an embedding of several disjoint analytic loops, but we will focus on one to simplify notation. A basic (nongeneric) example is the case when $\widehat{\Sigma}$ is a double and $c$ is a parameterization of $S$, the fixed point set of the involution $R$.

Let $\mathcal{O}$ denote the structure sheaf of $\widehat{\Sigma}$. There is an associated mapping

$$
E=E_{c, \mathcal{O}}: \operatorname{Hyp}\left(S^{1}, G\right) \rightarrow H^{1}\left(\mathcal{O}_{G}\right): \quad[g, h] \rightarrow E_{c}([g, h]),
$$

where $H^{1}\left(\mathcal{O}_{G}\right)$ denotes the set of isomorphism classes of holomorphic $G$-bundles on $\widehat{\Sigma}$. For an ordinary analytic $G$-valued loop $g, E$ maps the loop to the isomorphism class of the holomorphic $G$-bundle defined by using the loop as a holomorphic transition function in a tubular neighborhood of the image of $c$.

Remark A.1. Our convention for transition functions is the following. A (holomorphic) section of $E(g)$ (which one should think of as a frame) is a (holomorphic) function $s: \widehat{\Sigma} \backslash c \rightarrow G$ such that $s_{+} g^{-1}=s_{-}$, where $s_{+}\left(s_{-}\right)$is the restriction of $s$ to a sufficiently small annulus to the left (respectively, the right) of the oriented loop $c$. Consequently for an associated bundle $E(g) \times_{G} V$ a (holomorphic) section is represented by a (holomorphic) function $v: \widehat{\Sigma} \backslash c \rightarrow V$ such that $g v_{+}=v_{-}$.

With this convention, for scalar transition functions, the degree of a line bundle is the negative of the degree of a corresponding transition function, e.g., for differentials on $\mathbb{P}^{1}, d w=d z\left(-z^{-2}\right)$ $\left(s_{-}=(d w)\right.$ and $\left.s_{+}=(d z)\right)$; the degree of the canonical bundle is 2 , and the degree of the transition function $-z^{2}$ is -2 . 
The point is that $E$ can be extended naturally to hyperfunctions in the following way. The map $c$ extends uniquely to a holomorphic embedding $c:\{1-\epsilon<|z|<1+\epsilon\} \rightarrow \widehat{\Sigma}$ for some $\epsilon>0$. Given the pair $(g, h)$, we obtain a holomorphic bundle on $\widehat{\Sigma}$ by using $g$ as a transition function on an $\epsilon^{\prime}$-collar to the left of $c$ and $h$ as a transition function on an $\epsilon^{\prime}$-collar to the right of $c$, for some $\epsilon^{\prime}<\epsilon$, depending upon the pair $(g, h)$. The isomorphism class of this bundle is independent of the choice of $\epsilon^{\prime}$, and depends only upon $[g, h] \in \operatorname{Hyp}\left(S^{1}, G\right)$.

The basic properties of the mapping $E_{c}$ are summarized as follows.

\section{Proposition A.2.}

(a) If $\phi \in C^{\omega} \operatorname{Hom}\left(S^{1}\right)$, then the induced map

$$
\operatorname{Hyp}\left(S^{1}, G\right) \stackrel{\phi}{\rightarrow} \operatorname{Hyp}\left(S^{1}, G\right) \stackrel{E_{f}}{\rightarrow} H^{1}\left(\mathcal{O}_{G}\right)
$$

equals $E_{c \circ \phi^{-1}}$.

(b) There is a holomorphic action

$$
H^{0}\left(\widehat{\Sigma} \backslash c\left(S^{1}\right)\right) \times \operatorname{Hyp}\left(S^{1}, G\right) \rightarrow \operatorname{Hyp}\left(S^{1}, G\right): f,[g, h] \rightarrow\left[\left.f\right|_{S_{-}^{1}} g,\left.h f\right|_{S_{+}^{1}} ^{-1}\right] .
$$

(c) Inclusion and the mapping $E_{c}$ induce isomorphisms of sets

$$
H^{0}\left(S^{1}, G\right) / H^{0}(\overline{\widehat{\Sigma} \backslash c}, G) \rightarrow \operatorname{Hyp}\left(S^{1}, G\right) / H^{0}(\widehat{\Sigma} \backslash c) \rightarrow H^{1}\left(\mathcal{O}_{G}\right)
$$

where $\overline{\widehat{\Sigma} \backslash c}$ denotes the closed Riemann surface obtained by cutting along $c$ and adding two boundary components (see [16, Section 8.11]).

An alternate way to think about the projection $E_{c, \mathcal{O}}$ is as follows. Suppose that $(g, h)$ represents $[g, h] \in \operatorname{Hyp}\left(S^{1}, G\right)$. In the $C^{\infty}$ category the principal bundle defined using the transition functions $(g, h)$ is trivial. Therefore we can find smooth functions

$$
s: \widehat{\Sigma} \backslash c\left(S^{1}\right) \rightarrow G \quad \text { and } \quad s_{0}: S^{1 \epsilon} \rightarrow G
$$

(where $S^{1 \epsilon}$ is an $\epsilon$-collar neighborhood of $S^{1}$ in $\widehat{\Sigma}$, and the $\epsilon$-collar will depend upon $(g, h)$ ) such that

$$
s_{-}=g s_{0} \quad \text { and } \quad s_{0}=h s_{+}
$$

on an $\epsilon$ collar to the left (respectively, the right) of $S^{1}$. If $s$ is replaced by $s^{\prime}$, then there exists $f \in C^{\infty}(\widehat{\Sigma}, G)$ such that $s^{\prime}=s f$, i.e., $s_{-}^{\prime}=s_{-} f$ and so on with + and 0 in place of - .

Define $a=s^{-1} \bar{\partial} s$. Then the gauge equivalence class of $a$ depends only upon $[g, h]$. For if we change $g, h$ to $g_{-} g g_{0}, g_{0}^{-1} h g_{+}$, where $g_{ \pm}$is holomorphic in $\widehat{\Sigma}^{ \pm}$, and $g_{0}$ is holomorphic in $S^{1 \epsilon}$, then the choice of $s$ is modified in an obvious way, and this does not change $a$, and when $s \rightarrow s^{\prime}=s f$, then $a \rightarrow f^{-1} a f+f^{-1} \bar{\partial} f$ (a gauge transformation). Thus we obtain a well-defined map

$$
\operatorname{Hyp}\left(S^{1}, G\right) \rightarrow \Omega^{0,1}(\widehat{\Sigma}, \mathfrak{g}) / C^{\infty}(\widehat{\Sigma}, G)=H^{1}\left(\mathcal{O}_{G}\right) .
$$

\section{A.4 The image of $L K$}

Given a simple loop $c$ on a closed Riemann surface $\widehat{\Sigma}, H^{0}\left(S^{1}, G\right)$ maps onto the set of all isomorphism classes of holomorphic $G$ bundles on $\widehat{\Sigma}$. The main point of this subsection is to show that, assuming $K$ is simply connected, the subgroup of $K$ valued loops also maps onto the set of all isomorphism classes of holomorphic bundles.

We first explain why this is true generically, but with critical exceptions, for non-simply connected $K$. 
Proposition A.3. Suppose $K=\mathbb{T}$. The map from circle valued loops (of degree zero) to isomorphism classes of holomorphic line bundles (of degree zero),

$$
E_{c}:(L \mathbb{T})_{0} \rightarrow \operatorname{Jac}(\widehat{\Sigma})
$$

is not surjective if and only if $c$ is a straight line with respect to the flat geometry defined by some holomorphic differential.

Proof. We must determine when the connecting map, as in (2.1),

$$
C^{\omega}(\operatorname{Im}(c) ; \mathbb{R}) \rightarrow H^{0,1}(\hat{\Sigma}): f \rightarrow \bar{\partial} F
$$

is surjective, where in the $C^{\infty}$ category, $f=F_{+}-F_{-}, F$ is a smooth function on the complement of $c$, with limits $F_{ \pm}$from the left (right, respectively) of $c$. This map fails to be surjective if and only if there exists a nonzero holomorphic differential $\omega$ such that $\operatorname{Re}\left(\int_{\hat{\Sigma}} \bar{\partial} F \wedge \omega\right)=0$ for all $f$, i.e., there is a nonzero real harmonic one form $\eta=\omega+\bar{\omega}$ such that $\int_{c} f \eta=0$ for all real functions on $c$, which is equivalent to saying that the pullback of $\eta$ to $c$ vanishes. Locally $\omega=w d z$ and the corresponding flat metric (with conical singularities at the zeros of $\omega$ ) is $|d W|^{2}=|w|^{2}|d z|^{2}$. The vanishing of the pullback of $\operatorname{Re}(d W)$ along $c$ is equivalent to saying that $c$ is a straight line.

Suppose that $\widehat{\Sigma}$ is a double of $\Sigma$, a compact Riemann surface with boundary, as in the text, and $\operatorname{Im}(c)=S$. The Jacobian of $\widehat{\Sigma}$ fits into a short exact sequence

$$
0 \rightarrow H^{1}(\widehat{\Sigma}, 2 \pi \mathbb{Z}) \rightarrow H^{1}(\widehat{\Sigma}, \mathbb{R}) \rightarrow \operatorname{Jac}(\widehat{\Sigma}) \rightarrow 0 .
$$

$R$ acts equivariantly on this sequence, using pullback. $R$ also acts equivariantly on the projection $(L T)_{0} \rightarrow \operatorname{Jac}(\widehat{\Sigma})$, where the action on transition functions along $S$ is given by

$$
g \rightarrow g^{*}:=\left(R^{*} g\right)^{-1} .
$$

In this case the image consists of line bundles having an antiholomorphic reflection symmetry compatible with $R$, and these correspond to cohomology classes which are fixed by $R$. This is precisely the kind of (sub-line bundle) degeneracy that we encountered in Theorem 1.3.

Suppose now that $K$ is simply connected. Given $R$ and the involution $\tau$ defining $K$ as a real form of $G$, there is an involution of $\Omega^{0}(S, G)$ which fixes $\Omega^{0}(S, K): g \rightarrow \tau(g \circ R)$. This does not have an extension to $\operatorname{Hyp}(S, G)$ (in sharp contrast to the abelian case). For in the nonabelian case this involution is not compatible with the action in part (b) of Proposition A.2. Consequently there does not exist a real form for isomorphism classes of $G$ bundles on $\widehat{\Sigma}$ which would confine the image of $\Omega^{0}(S, K)$, as in the abelian case.

In the nonabelian case we conjecture that the ordered product map

$$
H^{0}\left(\Sigma^{*}, G\right) \times \Omega^{0}(S, K) \times H^{0}(\Sigma, G) \rightarrow \Omega^{0}(S, G)
$$

is surjective. Our objective is to explain why this is plausible.

For a group $H$ use left translation to identify $H \times \mathfrak{h}$ with $T H$. With this convention at the point $\left(g_{-}, k, g_{+}\right)$, the derivative of the ordered product mapping is given by the formula

$$
\begin{aligned}
& H^{0}\left(\Sigma^{*}, \mathfrak{g}\right) \times \Omega^{0}(S, \mathfrak{k}) \times H^{0}(\Sigma, \mathfrak{g}) \rightarrow \Omega^{0}(S, \mathfrak{g}), \\
& \left.\left(X_{-}, x, X_{+}\right) \rightarrow \frac{d}{d t}\right|_{t=0}\left(g_{+}^{-1} k^{-1} g_{-}^{-1} g_{-} e^{t X_{-}} k e^{t x} g_{+} e^{t X_{+}}\right)=g_{+}^{-1} k^{-1} X_{-} k g_{+}+g_{+}^{-1} x g_{+}+X_{+} .
\end{aligned}
$$

Lemma A.4. The derivative of the ordered product map at $\left(g_{-}, k, g_{+}\right)$is critical (i.e., not surjective) if $k$ is degenerate in the sense that

$$
\cap_{z \in S^{1}} \operatorname{ker}(\operatorname{Ad}(k(z)) \neq\{0\} .
$$




\section{Remark A.5.}

(a) Conjecturally the converse to the lemma holds.

(b) In a heuristic way, this is telling us when the map

$$
\Omega^{0}(S, K) \rightarrow H^{0}\left(\Sigma^{*}, G\right) \backslash \Omega^{0}(S, G) / H^{0}(\Sigma, G)
$$

is regular, i.e., has a surjective derivative.

Proof. The derivative of the ordered product map at $\left(g_{-}, k, g_{+}\right)$is surjective if and only if the the map

$$
\begin{aligned}
& H^{0}\left(\Sigma^{*}, \mathfrak{g}\right) \times \Omega^{0}(S, \mathfrak{k}) \times H^{0}(\Sigma, \mathfrak{g}) \rightarrow \Omega^{0}(S, \mathfrak{g}), \\
& \left(X_{-}, x, X_{+}\right) \rightarrow X_{-}+x+k X_{+} k^{-1}
\end{aligned}
$$

is surjective if and only if the dual map is one to one. To compute the dual map, we identify the dual of $\Omega^{0}(S, \mathfrak{g})$ with itself using the invariant form. The dual map is then given by

$$
\begin{aligned}
& \Omega^{0}(S, \mathfrak{g}) \rightarrow H^{0}\left(\Sigma^{*}, \mathfrak{g}\right) \times \Omega^{0}(S, \mathfrak{k}) \times H^{0}(\Sigma, \mathfrak{g}), \\
& \phi \rightarrow\left(\phi_{-}, \phi_{L \mathfrak{k}},\left(k^{-1} \phi k\right)_{+}\right) .
\end{aligned}
$$

This map is one to one if and only if the map (with finite-dimensional domain)

$$
\left\{\phi_{0}: \phi_{0}^{*}=\phi_{0}\right\} \rightarrow H^{0}(\Sigma, \mathfrak{g}): \phi_{0} \rightarrow\left(k^{-1}\left(\phi_{0}+\phi_{0}^{*}\right) k\right)_{+}
$$

is one to one (here $\left\{\phi_{0}\right\}$ is the vector space of zero modes with values in $\mathfrak{g}$ ). If $k$ is degenerate as in the statement of the lemma, then given $X \in \operatorname{ker}\left(\operatorname{Ad}(k(z))\right.$ for all $z \in S^{1}$, and any zero scalar zero mode $\chi_{0}$ satisfying $\chi_{0}=\chi_{0}^{*}, \phi_{0}=\chi_{0} X$ is in the kernel of (A.4).

Conversely suppose $\phi_{0}$ is in the kernel of (A.4). Then

$$
\psi:=k^{-1} \phi_{0} k=\psi_{0}+\psi_{-}=\psi^{*}=\psi_{0}^{*}+\psi_{+}
$$

Consequently $k^{-1} \phi_{0} k$ is another zero mode satisfying $\psi_{0}=\psi_{0}^{*}$. This would seem to force $k$ to be degenerate, but this is not entirely clear.

At the point $(1,1,1)$, the derivative is the sum mapping

$$
H^{0}\left(\Sigma^{*}, \mathfrak{g}\right) \times \Omega^{0}(S, \mathfrak{k}) \times H^{0}(\Sigma, \mathfrak{g}) \rightarrow \Omega^{0}(S, \mathfrak{g})
$$

The image of this sum is proper and equal to

$$
\Omega^{0}(S, \mathfrak{k})+H^{0}(\Sigma, \mathfrak{g})
$$

However at a generic point, $\left(g_{-}, k, g_{+}\right)$, because of noncommutativity, the derivative is surjective. At these points the map is locally open.

This derivative calculation strongly supports the conjecture, but it is not clear how to parlay this into a proof. 


\section{References}

[1] Basor E., Pickrell D., Loops in SU(2), Riemann surfaces, and factorization, II: Examples, in progress.

[2] Bott R., Stable bundles revisited, in Surveys in Differential Geometry (Cambridge, MA, 1990), Lehigh University, Bethlehem, PA, 1991, 1-18.

[3] Böttcher A., Silbermann B., Analysis of Toeplitz operators, Springer-Verlag, Berlin, 1990.

[4] Clancey K.F., Gohberg I., Factorization of matrix functions and singular integral operators, Operator Theory: Advances and Applications, Vol. 3, Birkhäuser Verlag, Basel - Boston, Mass., 1981.

[5] Gohberg I., Krupnik N., Einführung in die Theorie der eindimensionalen singulären Integraloperatoren, Mathematische Reihe, Vol. 63, Birkhäuser Verlag, Basel - Boston, Mass., 1979.

[6] Griffiths P., Harris J., Principles of algebraic geometry, Pure and Applied Mathematics, John Wiley \& Sons, New York, 1978.

[7] Grothendieck A., Sur la classification des fibrés holomorphes sur la sphère de Riemann, Amer. J. Math. 79 (1957), 121-138.

[8] Hawley N.S., Schiffer M., Half-order differentials on Riemann surfaces, Acta Math. 115 (1966), $199-236$.

[9] Helgason S., Groups and geometric analysis. Integral geometry, invariant differential operators, and spherical functions, Pure and Applied Mathematics, Vol. 113, Academic Press, Inc., Orlando, FL, 1984.

[10] Helton J.W., Howe R.E., Integral operators: commutators, traces, index and homology, in Proceedings of a Conference Operator Theory (Dalhousie Univ., Halifax, N.S., 1973), Lecture Notes in Math., Vol. 345, Springer, Berlin, 1973, 141-209.

[11] Krichever I.M., Novikov S.P., Virasoro-Gelfand-Fuks type algebras, Riemann surfaces, operator's theory of closed strings, J. Geom. Phys. 5 (1989), 631-661.

[12] Peller V.V., Hankel operators and their applications, Springer Monographs in Mathematics, Springer-Verlag, New York, 2003.

[13] Pickrell D., Invariant measures for unitary forms of Kac-Moody groups, Mem. Amer. Math. Soc. 146 (2000), 144 pages, funct-an/9510005.

[14] Pickrell D., Homogeneous Poisson structures on loop spaces of symmetric spaces, SIGMA 4 (2008), 069, 33 pages, arXiv:0801.3277.

[15] Pickrell D., Loops in SU(2) and factorization, J. Funct. Anal. 260 (2011), 2191-2221, arXiv:0903.4983.

[16] Pressley A., Segal G., Loop groups, Oxford Mathematical Monographs, The Clarendon Press, Oxford University Press, New York, 1986, oxford Science Publications.

[17] Ramanathan A., Stable principal bundles on a compact Riemann surface, Math. Ann. 213 (1975), 129-152.

[18] Rodin Y.L., The Riemann boundary value problem on closed Riemann surfaces and integrable systems, Phys. D 24 (1987), 1-53.

[19] Sato M., Theory of hyperfunctions. I, J. Fac. Sci. Univ. Tokyo. Sect. I 8 (1959), 139-193.

[20] Segal G., The definition of conformal field theory, in Topology, Geometry and Quantum Field Theory, London Mathematical Society Lecture Note Series, Vol. 308, Cambridge University Press, Cambridge, 2004, $421-577$.

[21] Widom H., Asymptotic behavior of block Toeplitz matrices and determinants. II, Adv. Math. 21 (1976), $1-29$. 